\title{
GENERALIZED HÉNON MAP AND BIFURCATIONS OF HOMOCLINIC TANGENCIES
}

\author{
V.S. GONCHENKO*, YU.A. KUZNETSOV ${ }^{\dagger}$, AND H.G.E. MEIJER ${ }^{\dagger}$
}

\begin{abstract}
We study two-parameter bifurcation diagrams of the generalized Hénon map (GHM), that is known to describe dynamics of iterated maps near homoclinic and heteroclinic tangencies. We prove the nondegeneracy of codim 2 bifurcations of fixed points of GHM analytically and compute its various global and local bifurcation curves numerically. Special attention is given to the interpretation of the results and their application to the analysis of bifurcations of the homoclinic tangency of a neutral saddle in two-parameter families of planar diffeomorphisms. In particular, an infinite cascade of homoclinic tangencies of neutral saddle cycles is shown to exist near the homoclinic tangency of the primary neutral saddle.
\end{abstract}

Key words. Homoclinic tangencies, Hénon map, normal forms, numerical continuation.

AMS subject classifications. 37G10(34C23,37G20)

1. Introduction. The real planar map

$$
\left(\begin{array}{c}
X \\
Y
\end{array}\right) \mapsto\left(\begin{array}{c}
1+Y-a X^{2} \\
b X
\end{array}\right)
$$

was first introduced by Hénon [19] as a planar diffeomorphism that mimics essential stretching and folding properties of the Poincaré map of the Lorenz system and has a strange attractor. It was also mentioned in [19], that any quadratic planar map with constant Jacobian can be put into the canonical form (1.1) by a linear coordinate transformation. Making another linear transformation, we can write $(1.1)$ as

$$
\left(\begin{array}{l}
x \\
y
\end{array}\right) \mapsto\left(\begin{array}{c}
y \\
\alpha-\beta x-y^{2}
\end{array}\right),
$$

which is called the standard Hénon map in what follows. Since the late 1970s, the standard Hénon map served as an important but artificial example to illustrate many analytical results and numerical techniques of dynamical systems theory.

It is remarkable that the map (1.2) was earlier derived by Gavrilov and Shilnikov in [8, 9] as the principle part of the Poincaré maps near the non-transverse homoclinic orbit to a saddle fixed point, where its stable and unstable invariant manifolds have quadratic tangency. This phenomenon has codimension one and is called the homoclinic tangency. Denote by $\lambda$ and $\gamma$ the eigenvalues of the saddle fixed point, so that $0<|\lambda|<1<|\gamma|$. If the saddle quantity $\sigma=|\lambda \gamma|<1$, a one-parameter unfolding of such a singularity leads to the standard Hénon map (1.2) with $\beta_{k}=O\left(\sigma^{-k}\right)$, where $k$ enumerates shrinking definition strips of the Poincaré maps near the critical homoclinic orbit (see Appendix). Thus, the appearing Hénon maps in this case have small Jacobians: $\beta_{k} \rightarrow 0$ as $k \rightarrow \infty$. If $\sigma>1$, a similar result holds for the inverse Poincaré maps.

The standard Hénon map and related problems giving rise to Smale's horseshoe have been the object of many studies. The references can be found, for example, in [26].

Many details on the bifurcation diagram of $(1.2)$ in the $(\beta, \alpha)$-plane have been obtained by Mira [25]. In Figure 1.1, principle bifurcation curves of (1.2) recomputed with CONTENT [22] are shown, which constitute a small part of the bifurcation set described in [25]. A "backbone" of the bifurcation diagram is formed by three curves: $t^{(1)}$ corresponding to the existence of a fixed point with eigenvalue $1 ; f^{(1)}$ corresponding to the existence of a fixed point with eigenvalue -1 ; and $n^{(1)}$, where (1.2) has a fixed point with eigenvalues $e^{ \pm i \theta}, 0<\theta<\pi$. Crossing the curves $t^{(1)}$ or $f^{(1)}$ for $\beta \neq \pm 1$ results in nondegenerate fold or flip (period-doubling) bifurcations, respectively. In contrast, a bifurcation at $n^{(1)}$ is degenerate, since for $|\beta|=1$ the map (1.2) is

\footnotetext{
*Institute for Applied Mathematics and Cybernetics, 10 Ulyanova st., Nizhny Novgorod, 603005 Russia.

${ }^{\dagger}$ Mathematisch Instituut, Universiteit Utrecht, Budapestlaan 6, 3584 CD Utrecht, The Netherlands. All correspondence should be sent to Yu.A. Kuznetsov.
} 


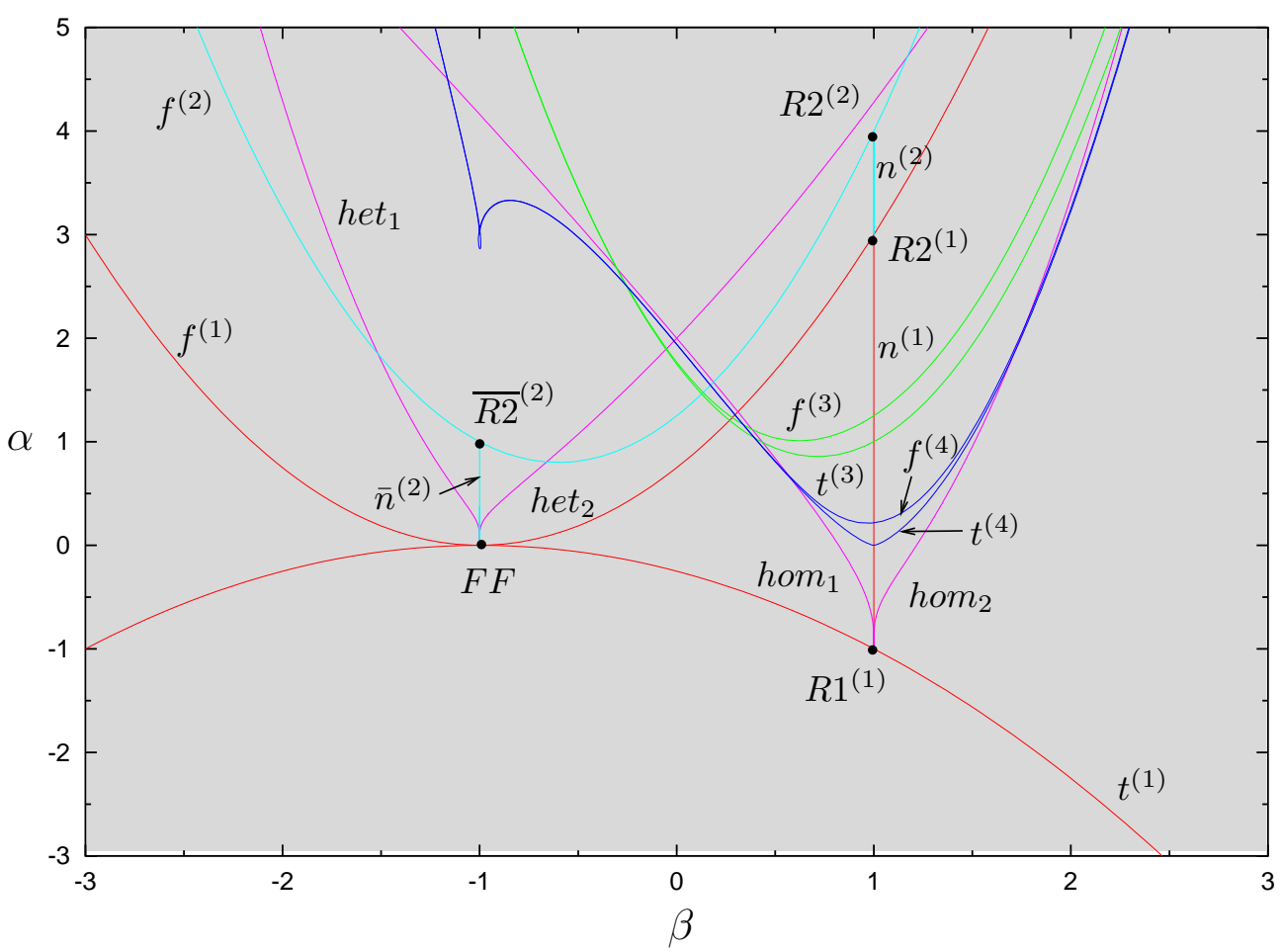

FIG. 1.1. Principle bifurcation curves of the standard Hénon map (1.2).

area-preserving (conservative). At intersections of these curves fixed points with double linear degeneracy exist: with eigenvalues \pm 1 (point $F F$ ), with a double eigenvalue 1 (point $R 1^{(1)}$ ); and with a double eigenvalue -1 (point $R 2^{(1)}$ ). These points are origins of other curves in the parameter plane corresponding to local and global bifurcations in (1.2). From $F F$ and $R 2^{(1)}$ the bifurcation lines $\bar{n}^{(2)}$ and $n^{(2)}$ start, respectively, where the standard Hénon map has a period-2 cycle with eigenvalues $e^{ \pm i \theta}, 0<\theta<\pi$. From $F F, R 1^{(1)}$, and $R 2^{(1)}$ other curves emanate, where (1.2) exhibits global bifurcations. Some of these curves are also shown in Figure 1.1. These are curves $h_{\text {om }}, 2$, where the stable and unstable invariant manifolds of a saddle fixed point are tangent (homoclinic tangency), and het $_{1,2}$, where a tangency of a stable manifold of one saddle with an

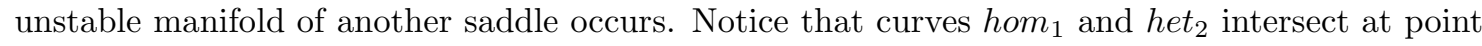
$(\beta, \alpha)=(0,2)$ on the $\alpha$-axis, along which the standard Hénon map is noninvertible and reduces to the scalar unimodular map $y \mapsto \alpha-y^{2}$. For $\alpha=2$ this scalar map indeed has an orbit connecting two fixed points and another orbit that is homoclinic to one of these fixed points. According to [25], the standard Hénon map (1.2) has an infinite number of bifurcation curves corresponding to fold $\left(t^{(k)}\right)$ and flip $\left(f^{(k)}\right)$ bifurcations of $k$-cycles with period $k>1$. Some of them are depicted in Figure 1.1. Several higher degeneracies on these curves were also reported and analyzed by Mira $[25]$.

Due to its conservative nature for $|\beta|=1$, the bifurcation diagram of the standard Hénon map (1.2) exhibits a number of highly degenerate features. This prevents applying Mira's results in homoclinic studies, where the standard Hénon map only approximates the actual Poincaré map.

In this paper, we study an extension of (1.2), namely the map

$$
\left(\begin{array}{c}
x \\
y
\end{array}\right) \mapsto\left(\begin{array}{c}
y \\
\alpha-\beta x-y^{2}+R x y+S y^{3}
\end{array}\right),
$$

where $R$ and $S$ are constants. This map is called the generalized Hénon map (GHM). For $R=$ $S=0$ it reduces to (1.2). Of course, one can add many different terms to the standard Hénon map to destroy its degeneracy. Our motivation to study this particular extension of (1.2) is that 
it appears in the bifurcation analysis of nontransversal homoclinic orbits and heteroclinic cycles of both codim 1 and 2 .

We focus on the following two codim 2 bifurcations of maps with homoclinic tangencies, which will be described in more detail in Section 2:

(1) the critical diffeomorphism in $\mathbb{R}^{2}$ has a neutral saddle $(\sigma=1)$ with a quadratic homoclinic tangency (see [10], [16] and [17]; the analogous case in $\mathbb{R}^{3}$ is considered in [18]);

(2) the critical diffeomorphism in $\mathbb{R}^{3}$ has a saddle with a generalized homoclinic tangency (i.e., the unstable manifold of the saddle has a quadratic tangency to its stable manifold but is nontransversal to leaves of the strong stable foliation in the stable manifold at the homoclinic points) (see [11]).

There are other global bifurcations of generic maps, where GHM appears naturally. If a diffeomorphism in $\mathbb{R}^{2}$ has two saddle fixed points $O_{1}$ and $O_{2}$ connected by two heteroclinic orbits, one of which is nontransversal (codim 1), then GHM appears as a rescaled first-return map when $\left(\sigma_{1}-1\right)\left(\sigma_{2}-1\right)<0$, where $\sigma_{i}$ is the saddle quantity of $O_{i}$ [14]. If a diffeomorphism in $\mathbb{R}^{3}$ has a codim 1 homoclinic tangency to a saddle-focus fixed point with eigenvalues $\nu_{1,2}=\lambda e^{ \pm i \varphi}, \nu_{3}=\gamma$, where $0<\lambda<1<\gamma, 0<\varphi<\pi$, and $\lambda^{2} \gamma<1$, then GHM appears when $\lambda \gamma>1[15]$.

GHM seems to play an important role in other homoclinic studies. From [7] it follows that the Poincaré map near a non-transverse homoclinic orbit to a saddle-saddle equilibrium in a threedimensional ODE is a smooth extension of the standard Hénon map (1.2) that can be reduced to GHM with $S=0$ and small $\beta$ and $R$. It is also known, that GHM with $|\beta|=1$ and $R=0$ appears in the analysis of two-dimensional area-preserving diffeomorphisms with homoclinic tangencies [13], and three-dimensional divergence-free ODEs with a homoclinic orbit to a saddle-focus equilibrium [5]. It seems that the appearance of GHM as a rescaled first-return map can be expected in other cases of homoclinic and heteroclinic tangencies, when the so-called "effective dimension of the problem" [27] can change.

This paper is organized as follows. In Section 2 we describe in detail two codim 2 homoclinic bifurcations, where the generalized Hénon map appears as a rescaled Poincaré map near the homoclinic orbit. Section 3 is devoted to analytical and numerical study of the bifurcation diagram of (1.3) in case $S=0$ and then in the general case $S \neq 0$. In Section 4 we discuss the correspondence between the bifurcations of the generalized Hénon map and those of the original diffeomorphisms with the codim 2 homoclinic tangencies. Some open problems are discussed in Section 5, while in Appendix we revisit the classical results of Gavrilov and Shilnikov using a modern scaling technique.

2. Homoclinic bifurcations and GHM. As mentioned in the Introduction, here we formulate two problems with homoclinic tangencies $[10,17]$ leading to the generalized Hénon map. Namely, we consider (1) a two-dimensional diffeomorphism with a homoclinic tangency of a neutral saddle; (2) a three-dimensional diffeomorphism with a generalized homoclinic tangency. In both cases the analysis leads to a two-parameter family $f_{\mu}, \mu=\left(\mu_{1}, \mu_{2}\right)^{T} \in \mathbb{R}^{2}$ of diffeomorphisms close to the diffeomorphism $f_{0}$. The first parameter $\mu_{1}$ in both cases is the splitting parameter, i.e., it measures the displacement of the stable and unstable manifolds. The second parameter $\mu_{2}$ is the deviation of the saddle quantity from 1 in the first case, and a characterization of the geometry in the second case.

We are interested in orbits located entirely in a small neighborhood $U$ of the critical homoclinic orbit. This neighborhood consists of a small neighborhood $U_{0}$ of the saddle fixed point and a finite number of small neighborhoods of points of the homoclinic orbit outside of $U_{0}$. We call a periodic orbit $p$-round if it lies entirely in $U$ and has exactly $p$ points within each of the small neighborhoods located outside $U_{0}$. Bifurcations of single-round orbits (1-round orbits) are studied below with the help of first-return (or Poincaré) maps.

2.1. Homoclinic tangency of a neutral saddle in $\mathbb{R}^{2}$. In this section we consider bifurcations in a generic two-parameter family $f_{\mu}$ of planar diffeomorphisms satisfying at $\mu=0$ the following conditions:

(A) $f_{0}$ has a saddle fixed point $O$ with eigenvalues $\gamma, \lambda$, such that $0<|\lambda|<1<|\gamma|$;

(B) the saddle quantity $\sigma \equiv|\lambda \gamma|=1$; 
(C) the invariant manifolds $W^{u}(O)$ and $W^{s}(O)$ have a quadratic tangency at points of a homoclinic orbit $\Gamma$ (see Figure 2.1).

2.1.1. Domains of definition. As usual in homoclinic studies, the Poincaré map is constructed as the composition of two maps: "local" - defined in $U_{0}$ and "global" - defined along the part of the homoclinic orbit in $U \backslash U_{0}$. In other words, single-round periodic orbits of period $k+n_{0}$ for all $k>\bar{k}$ (the meaning of $\bar{k}$ will be explained later) are fixed points of $f_{\mu}^{k+n_{0}} \equiv f_{\mu}^{n_{0}} \circ f_{\mu}^{k}$. In this construction, we will use a general representation of the global map $T_{1}=f_{\mu}^{n_{0}}$ (based only on the geometry of tangency) and a special form of the local map $T_{0}^{k}=f_{\mu}^{k}$, in which nonlinear terms are asymptotically small for $k \rightarrow \infty$ (see Appendix).

Let us consider two points of the critical homoclinic orbit $\Gamma$ in $U_{0}: M^{+}$on a local stable manifold and $M^{-}$on a local unstable manifold ${ }^{1}$. It is obvious that there is an integer $n_{0}$ such that $M^{+}=f_{0}^{n_{0}}\left(M^{-}\right)$.

Next we can choose two small neighborhoods: $\Pi^{+}$(of point $M^{+}$) and $\Pi^{-}$(of point $M^{-}$). Consider the forward images of $\Pi^{+}$under $f_{0}$. As is shown in Figure 2.2, there is some $\bar{k}$, such that for all $k \geq \bar{k}$ there is a "good" intersection of $f_{0}^{k}\left(\Pi^{+}\right)$and $\Pi^{-}$. "Good" means that $f_{0}^{k}\left(\Pi^{+}\right) \backslash \Pi^{-}$ consists of two components. Denote $f_{0}^{k}\left(\Pi^{+}\right) \cap \Pi^{-}$by $\sigma_{k}^{1}$. We can also iterate $\Pi^{-}$under $f_{0}^{-1}$ to obtain domains $\sigma_{k}^{0}=f_{0}^{-k}\left(\Pi^{-}\right) \cap \Pi^{+}$, which are the preimages of $\sigma_{k}^{1}$, i.e. $\sigma_{k}^{1}=f_{0}^{k}\left(\sigma_{k}^{0}\right)$. It is easy to see that the Poincaré map for the single-round $\left(k+n_{0}\right)$-periodic orbit is defined only in $\sigma_{k}^{0}$. Indeed, only $\sigma_{k}^{0}$ may consist of orbits which end up in $\Pi^{-}$after $k$ iterations $^{2}$.

Now we can define $T_{1} \circ T_{0}^{k}=f_{\mu}^{k+n_{0}}$ as the first-return map in such a strip for all $\mu$ with sufficiently small $\|\mu\|$. For details we refer to $[10,11]$. Here we give only a representation of the global map $T_{1}$, because it is used below:

$$
\begin{aligned}
\bar{x}_{0}-x^{+} \equiv & a x_{1}+b\left(y_{1}-y^{-}\right)+e_{20} x_{1}^{2}+e_{11} x_{1}\left(y_{1}-y^{-}\right)+e_{02}\left(y_{1}-y^{-}\right)^{2}+\cdots \\
\bar{y}_{0} \equiv & \mu_{1}+c x_{1}+d\left(y_{1}-y^{-}\right)^{2}+f_{20} x_{1}^{2}+f_{11} x_{1}\left(y_{1}-y^{-}\right)+ \\
& +f_{30} x_{1}^{3}+f_{21} x_{1}^{2}\left(y_{1}-y^{-}\right)+f_{12} x_{1}\left(y_{1}-y^{-}\right)^{2}+f_{03}\left(y_{1}-y^{-}\right)^{3}+\cdots
\end{aligned}
$$

Here all coefficients are smooth functions of $\mu$; moreover, $b c d \neq 0$. Note that the definition of the global map depends on $n_{0}$.

\footnotetext{
${ }^{1}$ In this neighborhood there are infinitely many such points - we choose any two of them

${ }^{2}$ If we consider 2-round periodic orbits the situation would be different, because then we should think about $i+j+2 n_{0}$-periodic orbits of type $(i, j)$. The 2 -round periodic orbit of type $(i, j)$ has $i$ points near the saddle, then $n_{0}$ points near the "global" part of the homoclinic orbit, then $j$ points near the saddle, and $n_{0}$ points near the homoclinic orbit. In this case, the domain of definition consists of two strips, $\sigma_{i}^{0}$ and $\sigma_{j}^{0}$.
}

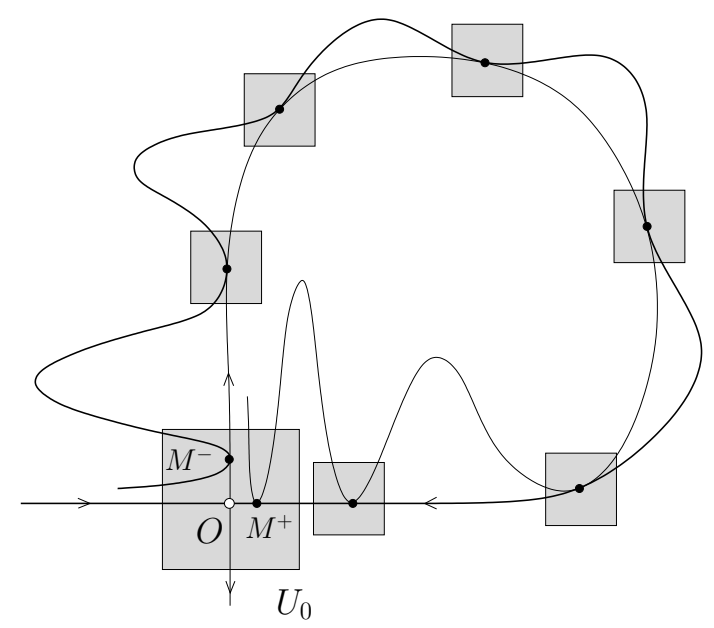

FIG. 2.1. Tangency of the stable and the unstable invariant manifolds. 


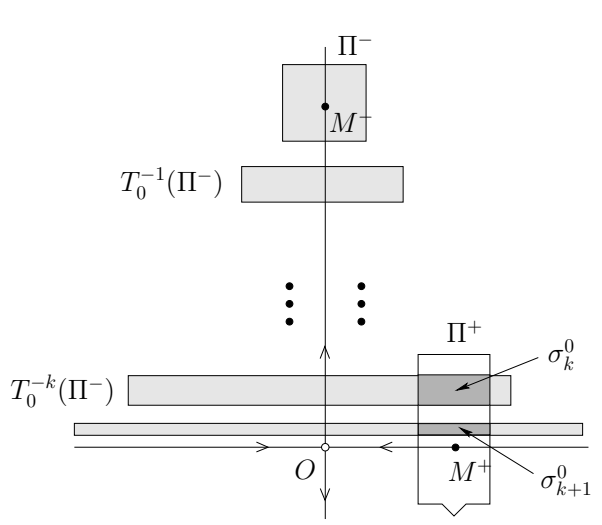

(a)

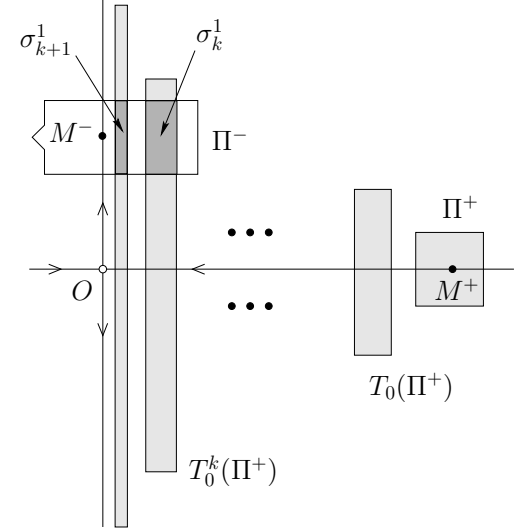

(b)

FIG. 2.2. Domains of definition: $\sigma_{k}^{1}=f_{0}^{k}\left(\sigma_{k}^{0}\right)$.

2.1.2. Rescaling results. Here we present only the results of the rescaling performed in [10]. The Poincaré map for any $k>\bar{k}$ can be reduced to the map

$$
\left\{\begin{array}{l}
\bar{X}=Y, \\
\bar{Y}=\alpha-\beta X-Y^{2}+R \lambda^{k} X Y+S \lambda^{k} Y^{3}+o\left(\lambda^{k}\right),
\end{array}\right.
$$

where $\lambda$ is the stable eigenvalue of the saddle, $\alpha, \beta, X, Y$ cover all finite values as $k \rightarrow \infty$, and $R, S$ depend on the coefficients of the global map (2.1), namely:

$$
R=2 a-\frac{b}{d} f_{11}-2 \frac{c}{d} e_{02}, \quad S=-\frac{b c}{d^{2}} f_{03} .
$$

There is the following correspondence between $(\alpha, \beta)$ and $\left(\mu_{1}, \mu_{2}\right)$ :

$$
\begin{aligned}
& \alpha=-d \gamma^{2 k}\left[\mu_{1}-\gamma^{-k}\left(y^{-}+\ldots\right)+c \lambda^{k}\left(x^{+}+\ldots\right)\right], \\
& \beta=-b c\left(1+\mu_{2}\right)^{k}(1+\ldots) .
\end{aligned}
$$

2.2. Generalized homoclinic tangency in $\mathbb{R}^{3}$. In this section we consider a generic twoparameter family $f_{\mu}$ of three-dimensional diffeomorphisms satisfying at $\mu=0$ the following conditions:

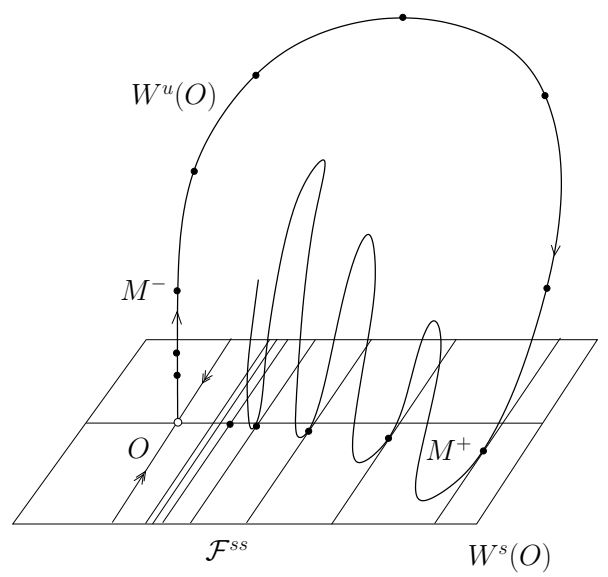

FIG. 2.3. Generalized homoclinic tangency 
(A) $f_{0}$ has a saddle fixed point $O$ with eigenvalues $\lambda_{1}, \lambda_{2}, \gamma$, such that $0<\left|\lambda_{2}\right|<\left|\lambda_{1}\right|<1<|\gamma|$;

(B) $\left|\lambda_{1} \gamma\right|>1,\left|\lambda_{2} \gamma\right|<1$;

(C) the invariant manifolds $W^{u}(O)$ and $W^{s}(O)$ have a quadratic tangency at points of a homoclinic orbit $\Gamma$ (see Figure 2.3);

(D) $T_{1}\left(P^{u e}\left(M^{-}\right)\right)$is transversal to $W_{l o c}^{s}$ at $M^{+}$but is tangent to $F^{s s}\left(M^{+}\right)$at $M^{+}$where $M^{+}, M^{-}$ are some points on local stable and local unstable manifolds respectively, $P^{u e}$ is the tangent plane to the extended unstable manifold at the point $M^{-}, T^{1}$ is the global map from a neighborhood of $M^{-}$to $M^{+}, F^{s s}\left(M^{+}\right)$is a leaf of the strong stable foliation containing $M^{+}{ }^{3}$.

2.2.1. Domains of definition. As in the planar case, we can choose two points on the homoclinic orbit in a sufficiently small neighborhood $U_{0}$ of the saddle fixed point: $M^{+}$on the local stable manifold, and $M^{-}$on the local unstable manifold. We also choose sufficiently small neighborhoods $\Pi^{+}$and $\Pi^{-}$of points $M^{+}$and $M^{-}$. We write $\bar{k}$ for the minimal $k$ such that $f_{0}^{k}\left(\Pi^{+}\right)$and $\Pi^{-}$have a "good" intersection. And we denote $\sigma_{k}^{1}=f_{0}^{k}\left(\Pi^{+}\right) \cap \Pi^{-}$and $\sigma_{k}^{0}=$ $f_{0}^{-k}\left(\Pi^{-}\right) \cap \Pi^{+}$. The picture is similar to Figure 2.2 but in three dimensions (with an additional contraction direction).

Also we need a representation for the global map $T_{1}$ :

$$
\begin{aligned}
\bar{x}_{1}-x_{1}^{+} & =a_{11} x_{1}+a_{12} x_{2}+\mu_{2}\left(y-y^{-}\right)+e_{02}\left(y-y^{-}\right)^{2}+\cdots, \\
\bar{x}_{2}-x_{2}^{+} & =a_{21} x_{1}+a_{22} x_{2}+b_{2}\left(y-y^{-}\right)+\cdots \\
\bar{y} & =\mu_{1}+c_{1} x_{1}+c_{2} x_{2}+d\left(y-y^{-}\right)^{2}+\cdots .
\end{aligned}
$$

Here all coefficients are smooth functions of $\mu$; moreover, $c_{1} d \neq 0$. As in the planar case, the definition of the global map depends on the choice of points $M^{ \pm}$.

2.2.2. Rescaling results. For a family $f_{\mu}$ of diffeomorphisms of $\mathbb{R}^{3}$ close to the diffeomorphism $f_{0}$ with the generalized homoclinic tangency the following result is valid [11]. The first-return map for $\left|\lambda_{1} \gamma\right|>1,\left|\lambda_{2} \gamma\right|<1$ and for any $k>\bar{k}$ can be reduced on some invariant 2 -dimensional manifold to the map

$$
\left\{\begin{array}{l}
\bar{X}=Y, \\
\bar{Y}=\alpha-\beta X-Y^{2}+R \lambda_{1}^{k} X Y+o\left(\lambda_{1}^{k}\right),
\end{array}\right.
$$

where

$$
R=2\left(a_{11}-\frac{e_{02} c_{1}}{d}\right)
$$

and

$$
\begin{aligned}
& \alpha=-d \gamma^{2 k}\left[\mu_{1}-\gamma^{-k}\left(y^{-}+\ldots\right)+\lambda^{k}\left(c_{1} x^{+}+\ldots\right)\right] \\
& \beta=-c_{1}\left(\mu_{2}+\ldots\right)\left(\lambda_{1} \gamma\right)^{k} .
\end{aligned}
$$

Note that in this case only the quadratic extra term appears (the term with $y^{3}$ exists but has order of $O\left(\gamma^{-k}\right)=o\left(\lambda^{k}\right)$ since $\left.\left|\lambda_{1} \gamma\right|>1\right)$.

When $\left|\lambda_{1} \gamma\right|>1$ and $\left|\lambda_{2} \gamma\right|>1$, another extension of the standard Hénon map appears [11].

3. Bifurcation diagrams of GHM. In this section we study the generalized Hénon map

$$
\left(\begin{array}{l}
x \\
y
\end{array}\right) \mapsto F_{\alpha, \beta}=\left(\begin{array}{c}
y \\
\alpha-\beta x-y^{2}+R x y+S y^{3}
\end{array}\right),
$$

where $R$ and $S$ are (not necessarily small) constants. Then we consider the limit case when $R$ and $S$ are substituted by $R \lambda^{k}$ and $S \lambda^{k}$, respectively. Since $|\lambda|<1$, these sequences tend to zero as $k \rightarrow \infty$.

\footnotetext{
${ }^{3}$ In this article we consider only one of the two cases treated in [11] (case I). Case II is similar.
} 
3.1. Codim 1 and 2 bifurcations of fixed points. This subsection is organized as follows. First we consider (3.1) with $S=0$. We derive the bifurcation curves for local codim 1 bifurcations and verify their nondegeneracy conditions. At the codim 2 points we compute the critical normal form coefficients. Thus we prove the nondegeneracy of these bifurcations for $R \neq 0$. Having this knowledge, we consider $S \neq 0$ and obtain similar results.

3.1.1. The Quadratic Extension $(S=0)$.

Proposition 3.1. The generalized Hénon map (3.1) with $S=0$ has the following codimension one bifurcations of fixed points:

Fold: If $R \neq 1$, there is a nondegenerate fold bifurcation for $\alpha=\frac{(\beta+1)^{2}}{4(R-1)}$ with the critical fixed point at $x=y=\frac{\beta+1}{2(R-1)}$.

Flip: If $R \neq 3$, there is a nondegenerate flip bifurcation for $\alpha=\frac{1}{4}(\beta+1)^{2}(3-R)$ with the critical fixed point at $x=y=\frac{\beta+1}{2}$.

Neimark-Sacker: If $R \neq 0,1,2$, there is a Neimark-Sacker bifurcation for

$$
\alpha=\frac{(\beta-1)(\beta-1+2 R)}{R^{2}}
$$

with the critical fixed point at $x=y=\frac{\beta-1}{R}$. It is defined for $\beta \in\left[\frac{2-3 R}{2-R}, \frac{2+R}{2-R}\right]$ when $R \in(0,2)$ and $\beta \in\left[\frac{2+R}{2-R}, \frac{2-3 R}{2-R}\right]$ when $R \notin[0,2]$. The bifurcation is nondegenerate away from strong resonances.

Proof. We write $D F$ for the Jacobian matrix of $F$ with respect to $(x, y)$. These curves follow easily from the fixed point equation, i.e. $F_{\alpha, \beta}(x)-x=0$, together with a condition on the multipliers. These conditions are given by the following equations are $\operatorname{det}(D F-I)=0$ (fold), $\operatorname{det}(D F+I)=0$ (flip) and $\operatorname{det}(D F)-1=0$ (NS). We have to exclude neutral saddles for the NS-bifurcation and therefore restrict to the interval as given above.

Then we have to check that these codim 1 bifurcations are nondegenerate. We use the formulas for the normal form coefficients from [21]. The bilinear form corresponding to the GHM with $S=0$ is

$$
B(x, y)=\left(\begin{array}{c}
0 \\
R\left(x_{1} y_{2}+y_{1} x_{2}\right)-2 x_{2} y_{2}
\end{array}\right)
$$

while the vectors

$$
\begin{gathered}
q_{\text {fold }}=\left(\begin{array}{c}
1 \\
1
\end{array}\right), \quad p_{\text {fold }}=\left(\begin{array}{c}
-\beta+\frac{(\beta+1) R}{2(R-1)} \\
1
\end{array}\right) \\
q_{f l i p}=\left(\begin{array}{c}
-1 \\
1
\end{array}\right), \quad p_{f l i p}=\left(\begin{array}{c}
\frac{1}{2}(\beta(2-R)-R) \\
1
\end{array}\right), \\
q_{n s}=\left(\frac{(\beta-1)(R-2)+\sqrt{(\beta-1)^{2}(R-2)^{2}-4 R^{2}}}{2 R}\right), p_{n s}=\left(\begin{array}{c}
-\frac{(\beta-1)(R-2)+\sqrt{(\beta-1)^{2}(R-2)^{2}-4 R^{2}}}{2 R} \\
1
\end{array}\right)
\end{gathered}
$$

satisfy the conditions:

$$
\begin{aligned}
& (D F) q_{\text {fold }}=q_{\text {fold }}, \quad(D F)^{T} p_{\text {fold }}=p_{\text {fold }}, \quad\left\langle p_{\text {fold }}, q_{\text {fold }}\right\rangle \neq 0, \\
& (D F) q_{\text {flip }}=q_{\text {flip }}, \quad(D F)^{T} p_{\text {flip }}=p_{\text {flip }}, \quad\left\langle p_{\text {flip }}, q_{\text {flip }}\right\rangle \neq 0, \\
& (D F) q_{n s}=e^{i \theta} q_{n s}, \quad(D F)^{T} p_{n s}=e^{i \theta} p_{n s}, \quad\left\langle p_{n s}, q_{n s}\right\rangle \neq 0 .
\end{aligned}
$$


We do not give intermediate calculations, but directly present the normal form coefficients

$$
\begin{aligned}
c_{\text {fold }} & =\frac{\left\langle p_{\text {fold }}, B\left(q_{\text {fold }}, q_{\text {fold }}\right)\right.}{\left\langle p_{\text {fold }}, q_{\text {fold }}\right\rangle}=\frac{4(1-R)^{2}}{\beta(2-R)-(2-3 R)} \\
c_{f l i p} & =\frac{\left\langle p_{\text {flip }}, B\left(q_{\text {flip }},(I-D F)^{-1} B\left(q_{\text {flip }}, q_{\text {flip }}\right)\right)\right\rangle}{2\left\langle p_{\text {flip }}, q_{\text {flip }}\right\rangle}=\frac{4(1+R)}{\beta(2-R)-(2+R)}, \\
c_{N S} & =\operatorname{Re}\left(\frac{e^{-i \theta}\left\langle p_{n s}, 2 B\left(q_{n s},(I-D F)^{-1} B\left(q_{n s}, \bar{q}_{n s}\right)\right)+B\left(\bar{q}_{n s},\left(e^{2 i \theta} I-D F\right)^{-1} B\left(q_{n s}, q_{n s}\right)\right)\right\rangle}{2\left\langle p_{n s}, q_{n s}\right\rangle}\right) \\
& =\frac{(1-R) R^{2}}{\beta(2-R)-(2-3 R)} .
\end{aligned}
$$

From the expressions above we see that for $R=-1,0,1,2$ the map is more degenerate.

Proposition 3.2. The generalized Hénon map (3.1) with $S=0, R \neq 0$, has the following codimension two bifurcations of fixed points:

Fold-flip There is a fold-flip bifurcation of the fixed point $x=y=0$ for $(\alpha, \beta)=(0,-1)$. The normal form coefficients defined in [23] are $a(0)=1-R, b(0)=1+R, c(0)=$ $\frac{3}{2}(1-R), d(0)=-\frac{1}{2}(5+3 R)$.

Resonance 1:1 There is a resonance $1: 1$ at $(\alpha, \beta)=\left(\frac{4(-1+R)}{(2-R)^{2}}, \frac{2-3 R}{2-R}\right)$ for the fixed point $x=y=\frac{\beta-1}{R}$. The normal form coefficient is $s=\operatorname{sgn}((1-R) R)$.

Resonance 1:2 There is a resonance 1:2 at $(\alpha, \beta)=\left(\frac{4(3-R)}{(2-R)^{2}}, \frac{2+R}{2-R}\right)$ for the fixed point $x=y=\frac{\beta-1}{R}$. The normal form coefficients are $C_{1}=-2(R+1)$ and $D_{1}=(1-R) R / 2$.

Resonance 1:3 There is a resonance $1: 3$ at $(\alpha, \beta)=\left(\frac{5-2 R}{(2-R)^{2}}, \frac{2}{2-R}\right)$ for the fixed point $x=y=\frac{\beta-1}{R}$. The normal form coefficient is $c=\frac{(1-R)(R-i \sqrt{3}(2+R))}{4\left(1+R+R^{2}\right)}$.

Resonance 1:4 There is a resonance 1:4 for the fixed point $x=y=0$ at $(\alpha, \beta)=(0,1)$. The normal form coefficient is $A(0)=\frac{R(1-R)+i\left(-2+3 R+R^{2}\right)}{\sqrt{\left(1+R^{2}\right)\left(4+4 R+2 R^{2}\right)}}$.

Proof. We calculate common points of the three bifurcation curves and find the first three codimension two bifurcations. It is easy to see that on the Neimark-Sacker bifurcation curve we encounter the strong resonances 1:3 and 1:4, while we move from the resonance 1:1 point to the 1:2 point. Solving for the eigenvalues, the parameter values for these bifurcations can be computed explicitly.

The fold-flip bifurcation was analyzed in [23]. For the strong resonance bifurcations we use the formulas from [21] without further references.

Fold-flip. Using the formulas from [23] with the critical eigenvectors

$$
q_{\text {fold }}=2 p_{\text {fold }}=\left(\begin{array}{l}
1 \\
1
\end{array}\right), \quad q_{\text {flip }}=2 p_{\text {flip }}=\left(\begin{array}{c}
1 \\
-1
\end{array}\right)
$$

we find for the truncated critical normal form

$$
\left(\begin{array}{c}
x_{1} \\
x_{2}
\end{array}\right) \mapsto\left(\begin{array}{c}
x_{1}+\frac{1}{2} a(0) x_{1}^{2}+\frac{1}{2} b(0) x_{2}^{2}+\frac{1}{6} c(0) x_{1}^{3}+\frac{1}{2} d(0) x_{1} x_{2}^{2} \\
-x_{2}+x_{1} x_{2}
\end{array}\right)
$$

the following coefficients

$$
a(0)=(1-R), \quad b(0)=(1+R), \quad c(0)=\frac{3}{2}(1-R), \quad d(0)=-\frac{1}{2}(5+3 R) .
$$

We see that, indeed, depending on $R$ the following cases occur.

$$
\begin{array}{cccc} 
& \operatorname{sign} a(0) & \operatorname{sign} b(0) \\
R<-1 & + & - & \text { case } 3 \\
-1<R<1 & + & + & \text { case } 1 \\
R>1 & - & + & \text { case } 2
\end{array}
$$


Next we calculate the critical coefficient determining the nondegeneracy of the $F F$ point (see [23]) to be $c_{N S}=R^{2}(1-R)$, meaning that a closed invariant curves coming from the Neimark-Sacker bifurcation of the period-2 cycle will be unstable for case 1 and stable for case 2 . Note that we excluded $R=0$ and $R=1$. For $(\alpha, \beta, R)=(0,-1,0)$ we actually have a codimension three point, since the coefficient $c_{N S}$ is then equal to zero.

Resonance 1:1 We shift the critical fixed point to the origin:

$$
\left(\begin{array}{l}
x \\
y
\end{array}\right) \mapsto\left(\begin{array}{l}
y \\
-x+2 y+R x y-y^{2}
\end{array}\right) .
$$

Bringing the linear part to the Jordan form, we get

$$
\left(\begin{array}{c}
x \\
y
\end{array}\right) \mapsto\left(\begin{array}{cc}
1 & 1 \\
0 & 1
\end{array}\right)\left(\begin{array}{c}
x \\
y
\end{array}\right)+\left(\begin{array}{c}
\frac{1}{2} a_{20} x^{2}+a_{11} x y+\frac{1}{2} a_{02} y^{2} \\
\frac{1}{2} b_{20} x^{2}+b_{11} x y+\frac{1}{2} b_{02} y^{2}
\end{array}\right),
$$

where $a_{20}=0, b_{20}=2(R-1)$ and $b_{11}=R-2$. This map is locally topologically equivalent to the time-one flow of the Bogdanov normal form

$$
\left\{\begin{array}{l}
\dot{\eta}_{1}=\eta_{2} \\
\dot{\eta}_{2}=\eta_{1}^{2}+s \eta_{1} \eta_{2}
\end{array}\right.
$$

Here the critical coefficient is given by $s=\operatorname{sgn}\left(b_{20}\left(a_{20}+b_{11}-b_{20}\right)\right)=\operatorname{sgn}((1-R) R)$.

Resonance 1:2 The shifted map can be written as

$$
\left(\begin{array}{c}
x \\
y
\end{array}\right) \mapsto\left(\begin{array}{c}
y \\
-x-2 y+R x y-y^{2}
\end{array}\right),
$$

while the time-one flow that approximates the second iterate of the normal form of this map is generated by

$$
\left(\begin{array}{c}
\dot{x} \\
\dot{y}
\end{array}\right)=\left(\begin{array}{c}
y \\
C_{1}(0) x^{3}+D_{1}(0) x y^{2}
\end{array}\right) .
$$

where $C_{1}(0)=-2(1+R)$ and $D_{1}(0)=(1-R) R / 2$.

Resonance 1:3 Shifting the fixed point to the origin and introducing a complex variable, we can write the critical map as

$$
z \mapsto \mu z+\sum_{k+l \geq 2} \frac{1}{k ! l !} g_{k l} z^{k} \bar{z}^{l}
$$

where $\mu=e^{i 2 \pi / 3}$ and $g_{20}=-1-\frac{i}{\sqrt{3}}(1+2 R), g_{11}=-1-\frac{R}{2}, g_{02}=-(1+R)+\frac{i}{\sqrt{3}}(R-1)$. The third iterate of the corresponding normal form can be approximated by the time-one flow of the equation

$$
\dot{z}=B_{1}(0) \bar{z}^{2}+C_{1}(0) z^{2} \bar{z}
$$

where

$$
B_{1}(0)=\frac{3 R}{2}+\frac{i}{2} \sqrt{3}(2+R), \quad C_{1}(0)=\frac{1}{2}(R-1)(-R+i \sqrt{3}(2+R))
$$

Since $B_{1}(0) \neq 0$ we can scale $C_{1}(0)$ to $c=\frac{C_{1}(0)}{\left|B_{1}(0)\right|^{2}}$. After some simplifications we find $c=$ $\frac{(R-1)(-R+i \sqrt{3}(2+R))}{4\left(1+R+R^{2}\right)}$.

Resonance 1:4 We apply the same procedure as above with $\mu=e^{i \pi / 2}$ and obtain $g_{20}=$ $\bar{g}_{02}=-1-i R$ and $g_{11}=-1$. The coefficients of the truncated critical normal form

$$
z \mapsto \mu z+C(0) z^{2} \bar{z}+D(0) \bar{z}^{3}
$$


are

$$
C(0)=\frac{1}{4}(1+i)\left((1-i)-(1-2 i) R-R^{2}\right), D(0)=\frac{1}{4}(1+i)\left((-1+i)+(1+2 i) R+R^{2}\right) .
$$

Since $D(0) \neq 0$ the flow approximating the 4 -th iterate of this map can be scaled to

$$
\dot{z}=A(0) z^{2} \bar{z}+\bar{z}^{3},
$$

where

$$
A(0)=\frac{-i C(0)}{|D(0)|}=\frac{R(1-R)+i\left(R^{2}+3 R-2\right)}{\sqrt{\left(1+R^{2}\right)\left(2 R^{2}+4 R+4\right)}} .
$$

We plot $A(0)$ as a parametric function of $R$ in Figure $3.1 \mathrm{\square}$

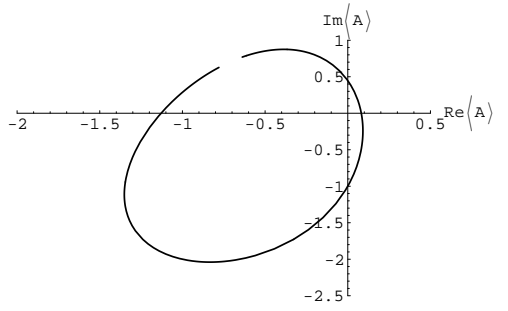

FIG. 3.1. The complex values of $A$ as a function of $R$.

3.1.2. The Cubic Extension $(S \neq 0)$.

Proposition 3.3. The generalized Hénon map (3.1) with $R, S \neq 0$ has the following codimension one bifurcations of fixed points:

Fold. There is a fold bifurcation at

$$
\alpha=\frac{2(1-R)^{3}+9(\beta+1)(1-R) S \pm 2\left((R-1)^{2}+3(\beta+1) S\right)^{3 / 2}}{27 S^{2}}
$$

of the fixed point

$$
x=y=\frac{(1-R) \pm \sqrt{(R-1)^{2}+3(\beta+1) S}}{3 S} .
$$

The fold normal form coefficient is given by

$$
c_{\text {fold }}=\frac{-6 S \sqrt{(1-R)^{2}+3(\beta+1) S}}{(3(\beta-1) S-R(1-R)) \pm R \sqrt{(1-R)^{2}+3(\beta+1) S}} .
$$

Flip. There is a flip bifurcation of the fixed point

$$
x=y=\frac{1 \pm \sqrt{1-3(\beta+1) S}}{3 S}
$$

at

$$
\alpha=\frac{2(1-3 R)+9(\beta+1)(R+1) S \pm 2(1-3 R+6(\beta+1) S) \sqrt{1-3(\beta+1) S}}{27 S^{2}} .
$$

The normal form coefficient is given by

$$
c_{f l i p}=\frac{3 S^{2}\left(-3+R-3 R^{2}+6(\beta-1) S \pm 7 R \sqrt{1-3(\beta+1) S}\right)}{(3(\beta-1) S+R(-1 \mp \sqrt{1-3(\beta+1) S)})(3(\beta+1) S+R(-1 \mp \sqrt{1-3(\beta+1) S}))} .
$$


Neimark-Sacker. There is a Neimark-Sacker bifurcation of the fixed point $x=y=\frac{\beta-1}{R}$ at

$$
\alpha=\frac{(\beta-1) R(\beta-1+2 R)-(\beta-1)^{3} S}{R^{3}} .
$$

for which we have

$$
c_{N S}=\frac{R^{2}((1-R) R-3 S(\beta-1)}{(\beta(2-R)-(2-3 R)) R-3(\beta-1)^{2} S} .
$$

Proof. The bifurcation curves follow from the same equations as in the previous proof. We should note that, unlike in the quadratic case, here the fold and flip curves consist of two branches which are connected. To prove the nondegeneracy we have to find the critical eigenvectors and higher order derivatives as before. We show computations for one branch only.

$$
\begin{gathered}
B(x, y)=\left(\begin{array}{c}
0 \\
R\left(x_{1} y_{2}+y_{1} x_{2}\right)-2 x_{2} y_{2}\left(R+\sqrt{(1-R)^{2}+3(\beta+1) S}\right)
\end{array}\right), \\
q_{\text {fold }}=\left(\begin{array}{l}
1 \\
1
\end{array}\right), \quad p_{\text {fold }}=\left(-\beta+\frac{R\left(-1+R+\sqrt{(1-R)^{2}+3(\beta+1) S}\right)}{1^{3 S}}\right) .
\end{gathered}
$$

With these definitions we calculate $c_{f o l d}=\langle p, B(q, q)\rangle /\langle p, q\rangle$ as given in the proposition on one branch. On the other it is similar. We proceed with the flip bifurcation.

$$
\begin{aligned}
& B(x, y)=\left(\begin{array}{c}
0 \\
R\left(x_{1} y_{2}+y_{1} x_{2}\right)-2 x_{2} y_{2} \sqrt{1-3(\beta+1) S}
\end{array}\right), C(x, y, z)=\left(\begin{array}{c}
0 \\
6 S x_{2} y_{2} z_{2}
\end{array}\right) \\
& q_{\text {flip }}=\left(\begin{array}{c}
-1 \\
1
\end{array}\right), \quad p_{\text {flip }}=\left(\beta+\frac{R(-1+\sqrt{1-3(\beta+1) S})}{1^{3 S}}\right)
\end{aligned}
$$

we calculate $c_{f l i p}=\left\langle p, C(q, q, q)-3 B\left(q,(A-I)^{-1} B(q, q)\right)\right\rangle / 6\langle p, q\rangle$ and find the expression on one branch.

For the Neimark-Sacker bifurcation we omit the intermediate calculations, but the coefficient $c_{N S}$ is obtained in the same way.

The above codimension one bifurcations are degenerate in several points. We treat them in two propositions. The cusp, generalized flip and generalized Neimark-Sacker are collected together, since they are determined by vanishing of the corresponding normal form coefficient. The fold-flip and the strong resonances fall into another group, since an extra condition on the multipliers is imposed.

Proposition 3.4. The generalized Hénon map (3.1) with $R, S \neq 0$ has the following codimension two bifurcations of fixed points:

Cusp. There is a cusp bifurcation of the fixed point $x=y=\frac{1-R}{3 S}$ at $\alpha=\frac{(R-1)^{3}}{27 S^{2}}$ and $\beta=-1-\frac{(R-1)^{2}}{3 S}$.

Generalized flip. With $\alpha, x, y$ defined in the previous PROPOSITION, the flip bifurcation is degenerate at

$$
\beta=-1+\frac{1}{24 S}\left(12-4 R-37 R^{2} \pm 7 R \sqrt{-8+8 R+25 R^{2}}\right) .
$$

Chenciner. The Neimark-Sacker bifurcation of the previous PROPOSITION is degenerate at $\alpha=\frac{(R-1)\left(R^{2}+R-2-18 S\right)}{27 S^{2}}, \beta=1+\frac{R(1-R)}{3 S}$, while the fixed point satisfies $x=y=\frac{1-R}{3 S}$. 
Proof. This follows easily from the expressions for the normal form coefficients at the codimension one bifurcations.

Proposition 3.5. The generalized Hénon map (3.1) with $R, S \neq 0$ has the following codimension two bifurcations of fixed points:

Fold-flip There is a fold-flip bifurcation of the fixed point $x=y=0$ at $\alpha=0$ and $\beta=-1$. There is another fold-flip bifurcation for $x=y=\frac{2-R}{3 S}$ and $\alpha=\frac{(R+1)(R-2)^{2}}{27 S^{2}}, \beta=$ $-1-\frac{R(R-2)}{3 S}$.

Resonance 1:1. For $\beta=1-\frac{R}{6 S}\left(R-2 \pm \sqrt{(R-2)^{2}+24 S}\right)$ there is a resonance 1:1.

Resonance 1:2. For $\beta=1-\frac{R}{6 S}\left(R-2 \pm \sqrt{(R-2)^{2}-24 S}\right)$ there is a resonance 1:2.

Resonance 1:3. For $\beta=1+\frac{R}{6 S}\left(R-2 \pm \sqrt{(R-2)^{2}-12 S}\right)$ there is a resonance 1:3.

Resonance 1:4. There is a resonance $1: 4$ at $\beta=1$ and $\beta=1-\frac{R(R-2)}{3 S}$.

It is possible that either the 1:1 or the 1:2 resonance does not exist. In the first case even the 1:3 resonance may not be present.

Proof. The eigenvalues can easily be computed along the codim 1 curves. Imposing an extra condition on them one finds the reported values for $\beta$ on the corresponding codim 1 curve. The nondegeneracy of the codim 2 points can also be checked.

3.2. Global bifurcations. It is known that the strong resonances and the fold-flip points are the origins of homoclinic and heteroclinic "horns". The boundaries of these horns correspond to primary homoclinic and heteroclinic tangencies, respectively. Here we use the algorithm from [4] to compute these boundary curves numerically.

Let us briefly explain the algorithm. Let $f(x)$ have a transverse homoclinic orbit at the fixed point $\xi$. We want to find the boundary, on which we have tangency. We approximate the homoclinic orbit by a finite number of points, say $n$. Let $q_{s}$ and $q_{u}$ be eigenvectors of the matrix $D F^{T}$, the transposed of the Jacobian, corresponding to the stable and unstable manifold respectively. Then we define the following algebraic system.

$$
\begin{aligned}
x_{i+1}-f\left(x_{i}\right) & =0 \quad \text { for } 1 \leq i \leq n-1, \\
\left\langle q_{s},\left(x_{1}-\xi\right)\right\rangle & =0 \\
\left\langle q_{u},\left(x_{n+1}-\xi\right\rangle\right. & =0 .
\end{aligned}
$$

The solution to this system can be continued in one parameter using CONTENT [22] and its limit point corresponds to a tangency of the stable and unstable manifolds. Continuation of such a limit point in CONTENT in two parameters using a standard defining system gives the boundary curves for homo- and heteroclinic structures.

To compute the stable and unstable manifolds of fixed points and cycles at fixed parameter values, we used the algorithms due to Krauskopf and Osinga [20] implemented in DsTools [3].

3.3. Small coefficients. As we have seen in Section 2, the Poincaré map for both codim 2 homoclinic tangency cases can be reduced to the map

$$
\begin{aligned}
& \bar{x}=y \\
& \bar{y}=\alpha-\beta x-y^{2}+R \lambda^{k} x y+S \lambda^{k} y^{3}+o\left(\lambda^{k}\right),
\end{aligned}
$$

where $|\lambda|<1$ is the stable eigenvalue of the saddle, $\alpha, \beta, x, y$ cover all finite values as $k \rightarrow \infty$. The closer we are to the neutral saddle, the higher $k$ we have to consider. Since $R$ and $S$ scale with $\lambda^{k}$, the case when $k$ is large corresponds to exponentially small coefficients $R$ and $S$. It follows from the previous text that the codim 1 bifurcation are nondegenerate away from the codim 2 bifurcations. If we use the parametric form of the Neimark-Sacker bifurcation curve,

$$
\alpha=\cos ^{2} \psi-2 \cos \psi, \quad \beta=1-R \cos \psi, \quad 0<\psi<\pi,
$$

we find

$$
c_{N S}=\frac{R}{4 \cos ^{2}\left(\frac{\psi}{2}\right)} \lambda^{k}+o\left(\lambda^{k}\right) .
$$


We find that for small $R$ the stability of the closed invariant curve emerging from the NS-bifurcation depends on the sign of $R$.

The codimension 2 bifurcations which occur only if $S \neq 0$, are at infinity and therefore inaccessible. For the codim 2 bifurcation we give the constant and linear terms in $\lambda^{k}$.

Fold-flip The normal form coefficients are

$$
\begin{gathered}
a(0)=1-R \lambda^{k}+o\left(\lambda^{k}\right), b(0)=1+R \lambda^{k}+o\left(\lambda^{k}\right), \\
c(0)=\frac{3}{2}\left(1-R \lambda^{k}\right)+3 S \lambda^{k}+o\left(\lambda^{k}\right), \quad d(0)=-\frac{1}{2}\left(5+3 R \lambda^{k}\right)+3 S \lambda^{k}+o\left(\lambda^{k}\right) .
\end{gathered}
$$

The critical coefficient $c_{N S}$ at the fold-flip point is ${ }^{4}$

$$
c_{N S}=\left(R^{2}+2 R S+8 s_{21}\right) \lambda^{2 k}+o\left(\lambda^{2 k}\right) .
$$

Resonance 1:1 The normal form coefficient $s$ has the same sign as $R \lambda^{k}$.

Resonance 1:2 The normal form coefficients are $C_{1}=-2+2(R-4 S) \lambda^{k}+o\left(\lambda^{k}\right)$ and $D_{1}=\frac{1}{2} R \lambda^{k}+o\left(\lambda^{k}\right)$.

Resonance 1:3 The nondegeneracy is given by the real part of the normal form coefficient $C_{1}=\frac{1}{6}\left(R \lambda^{k}+i \sqrt{3}\left(-2+(R-2 S) \lambda^{k}\right)\right)+o\left(\lambda^{k}\right)$.

Resonance 1:4 The normal form coefficient $A$ determining the bifurcation sequence is given by $A=-i+\left(\frac{1}{2}+2 i\right) R \lambda^{k}-4 i S \lambda^{k}+o\left(\lambda^{k}\right)$

We see immediately that when $R \rightarrow 0$, the bifurcations of the strong resonance become degenerate. For the fold-flip it follows that the Lyapunov coefficient for the Neimark-Sacker bifurcation of period 2 becomes degenerate if we do not consider $O\left(\lambda^{2 k}\right)$-terms.

3.4. Description of bifurcations. Here we describe the bifurcation diagram of the quadratic GHM for both positive and negative $R$. The bifurcations of the standard Hénon map remain as a backbone, compare Figure 1.1 and Figures 3.2, 3.3. However, from the previous sections, it follows that the bifurcations are nondegenerate for $R \neq 0$ and that the cubic term can be neglected if $S$ is small. We describe the local codim 2 bifurcations of low period of the generalized Hénon map, since these organize the parameter plane mostly. These points are also the origins of some global bifurcations, which are then addressed. All bifurcation curves are computed using CONTENT [22]. Below the $t^{(1)}$ curve there are no fixed points, and the dynamics are not interesting. On this curve there are two codim 2 points.

First we have the fold-flip point $F F$. For small $R$ this is case 1 of [23]. Near this bifurcation an unstable invariant closed circle appears and is destroyed. To show what happens, we take $\alpha=0.3$ and vary $\beta$, see Figures 3.4 and 3.5. From (1) to (3) we see the stable and unstable manifolds of the two saddle fixed points getting closer until they intersect. Furthermore we see that an Arnol'd tongue of period 12 is present around the period 2 fixed point. Going from (3) to (4) this orbit gains stability and an invariant closed curve is born. It consists of two disjoint sets and an iterate jumps from one set to the other. Close to $\bar{n}^{(2)}$ the closed invariant curve is smooth, but moving to (5) it becomes bigger and loses its smoothness. Eventually it "merges" with the stable manifold of the period 12 orbit and is destroyed. Then from (5) to (6) the period 12 orbits disappear through a fold bifurcation and the manifolds move away from each other.

The second codim 2 point $R 1^{(1)}$ on the $t^{(1)}$ curve is a 1:1 resonance. From this point a NeimarkSacker curve $n^{(1)}$ emerges. The corresponding invariant closed curve is stable for negative $R$ and unstable for positive $R$. The structure is completed with Arnol'd tongues and curves of homoclinic tangencies, see Figure 3.6.

The Neimark-Sacker bifurcation generates closed invariant curves, here we discuss their domain of existence. Possible destruction scenarios for a closed invariant curve inside the resonance tongue were studied theoretically in $[1,2,6]$. We consider here the lowest weak resonance, i.e. 1:5, which is located inside the homoclinic wedge of the 1:1 resonance. The bifurcation curves are shown in Figure 3.7, while the meaning of the hom-curves is explained in Figure 3.8. In the region 


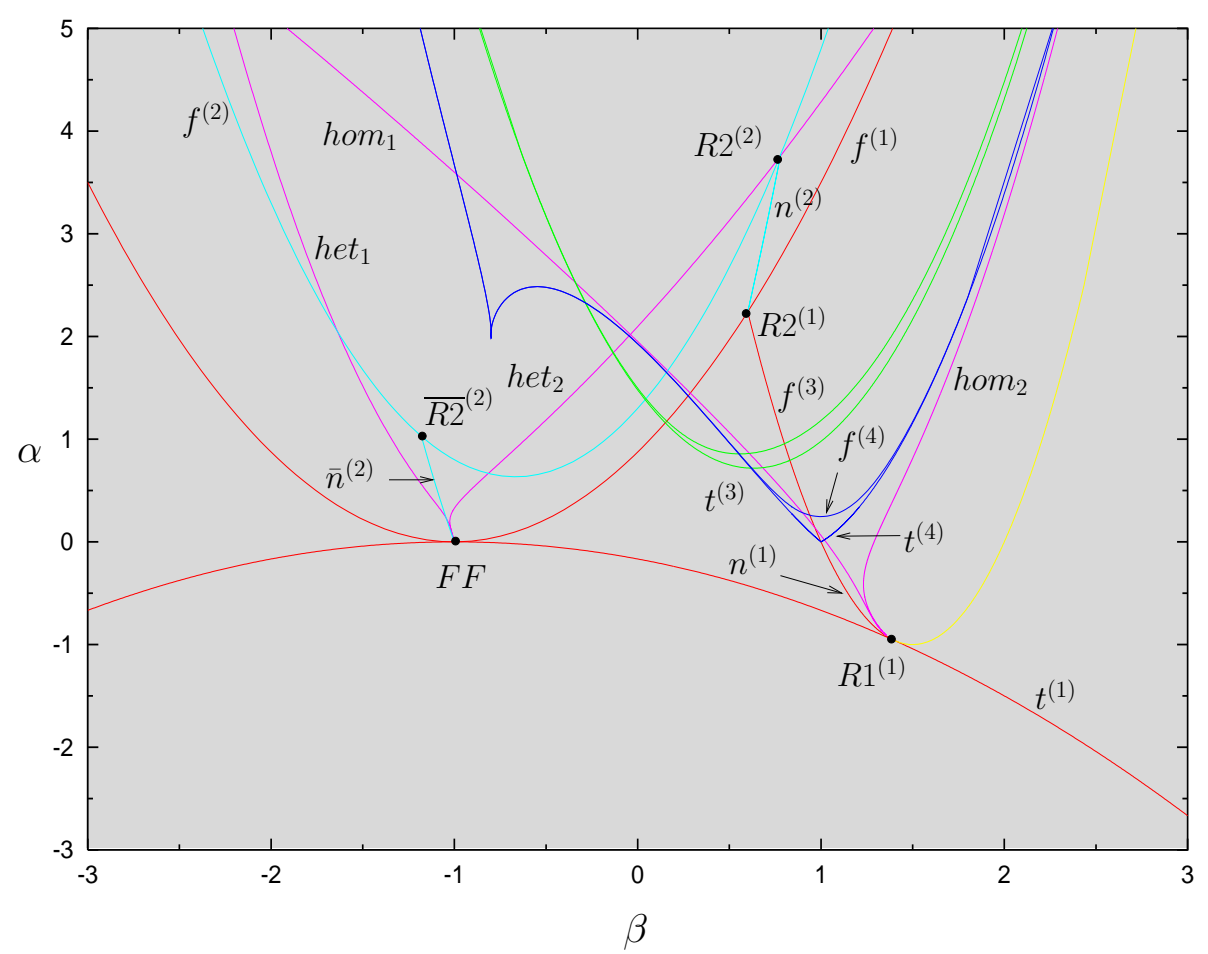

FiG. 3.2. Bifurcation diagram for the generalized Hénon map for $R=-0.5$.

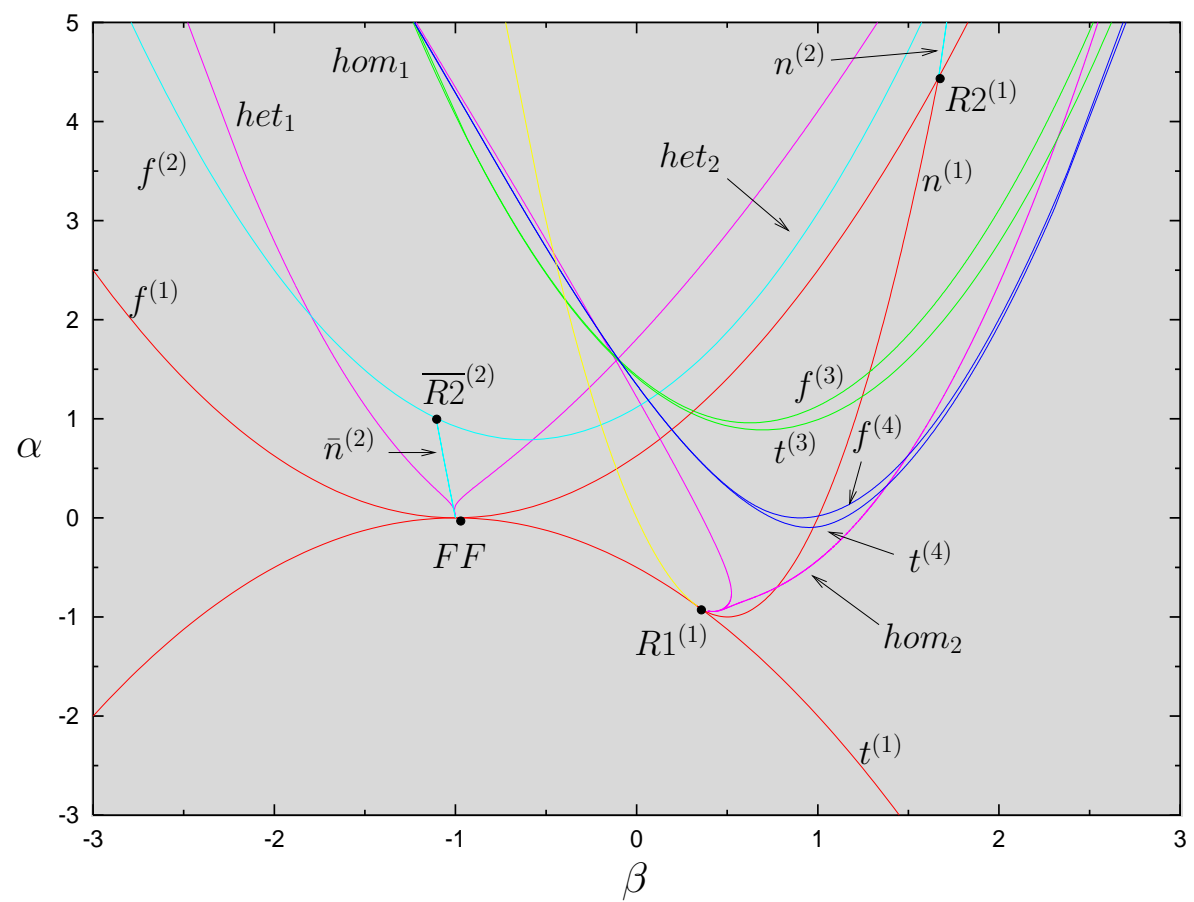

FIG. 3.3. Bifurcation diagram for the generalized Hénon map for $R=0.5$.

${ }^{4}$ We took every possible term of the order of $\lambda^{2 k}$ in the second equation of (3.29). The coefficient $s_{21}$ in front of the $x^{2} y$-term was the only relevant coefficient. 


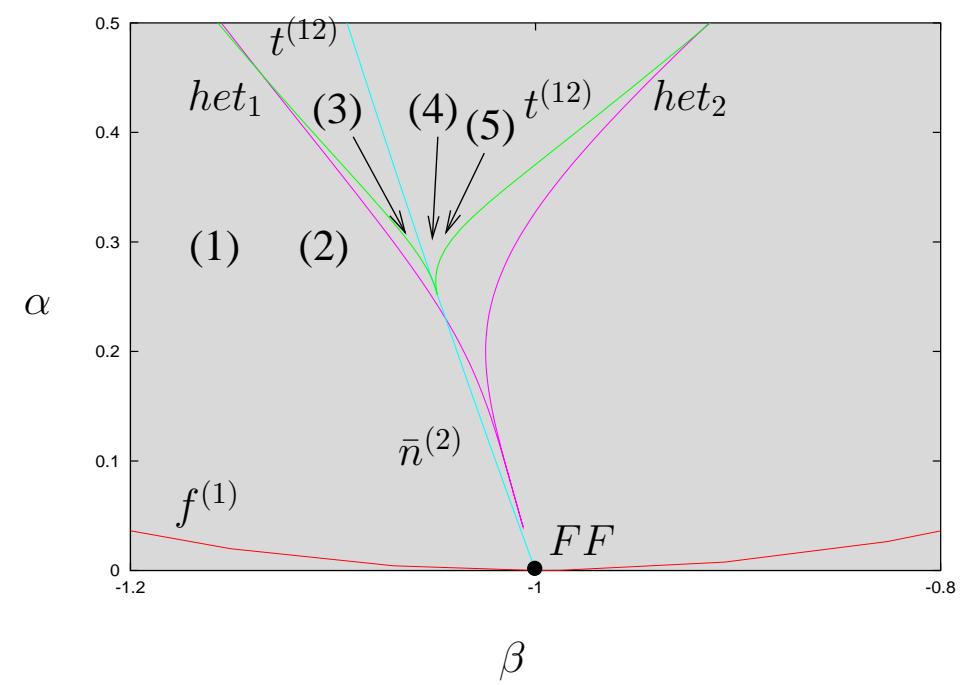

Fig. 3.4. Close up of the bifurcation diagram of the generalized Hénon map for $R=-0.5$ near the $F F$ point. Numbers correspond to phase portraits in Figure 3.5.

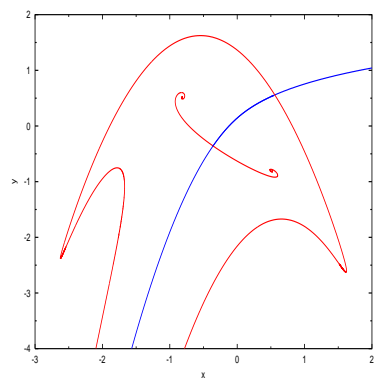

(1)

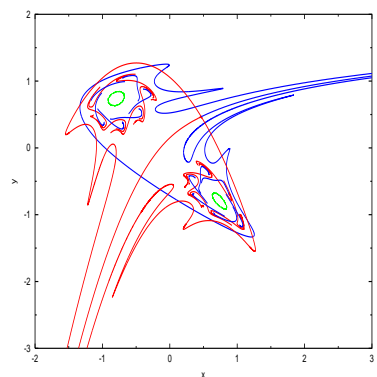

(4)

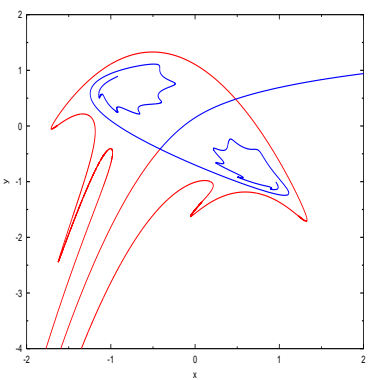

(2)

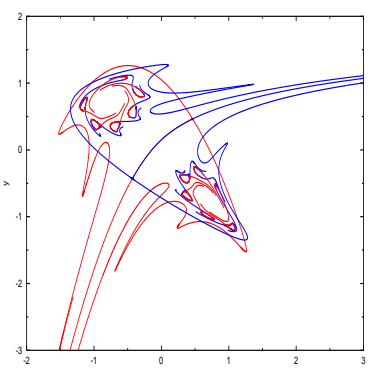

(5)

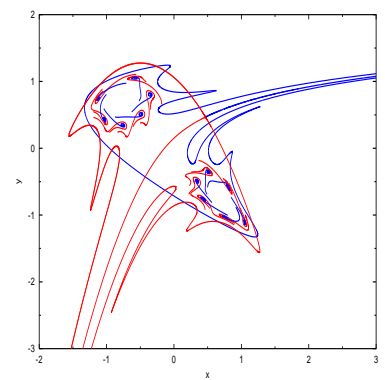

(3)

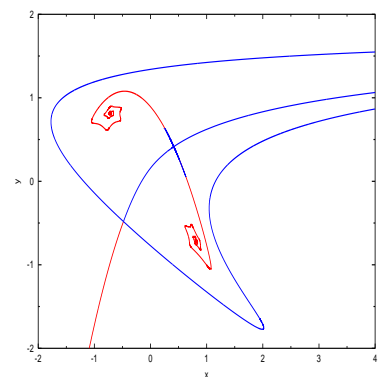

(6)

FIG. 3.5. Stable(blue) and unstable(red) manifolds near the fold-flip point. Parameter values are $R=$ $-0.5, \alpha=0.3$ and $\beta=-1.3(1),-1.1(2),-1.06(3),-1.057(4),-1.055(5),-0.9$ (6). The green object in (4) is the invariant closed circle.

bounded by $n^{(1)}$ and $h_{0} m_{i, i}$ a closed invariant curve exists, surrounded on the outside by saddles and nodes of period 5. Then there are two curves hom ${ }_{i, i}$ and hom $_{i, o}$. The distance between them becomes exponentially small if we move to the tip of the horn. In the region bounded by these two curves there are transversal homoclinic structures. Between curves hom $_{i, o}$ and hom $_{o, i}$ the invariant circle exists as the unstable manifold of a saddle together with a node. We remark that these two curves intersect below the flip $f^{(5)}$ and end up tangentially at the $t^{(5)}$-curves. If we move 


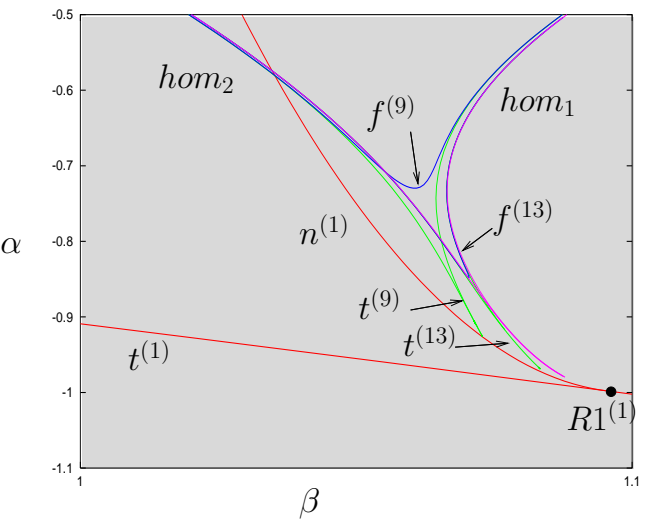

(a)

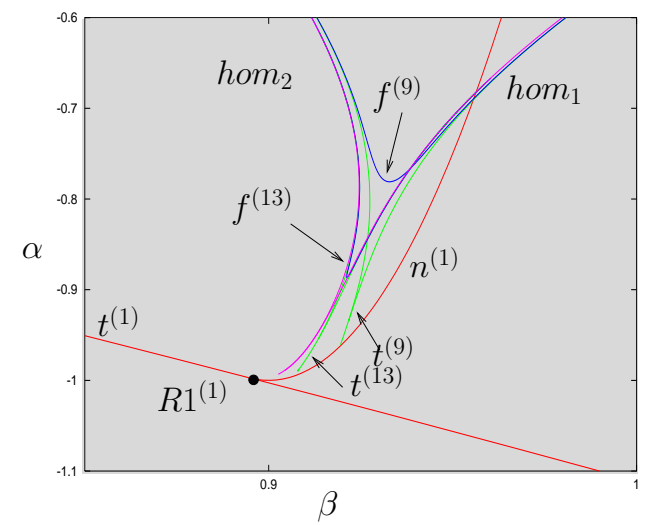

(b)

Fig. 3.6. Close-up near the 1:1 resonance for (a) $R=-0.1$, (b) $R=0.1$. Visible are fold and Neimark-Sacker of period 1(red), curves of homoclinic tangencies(yellow) and period 9(blue) and 13(green/pink) Arnol'd tongues.

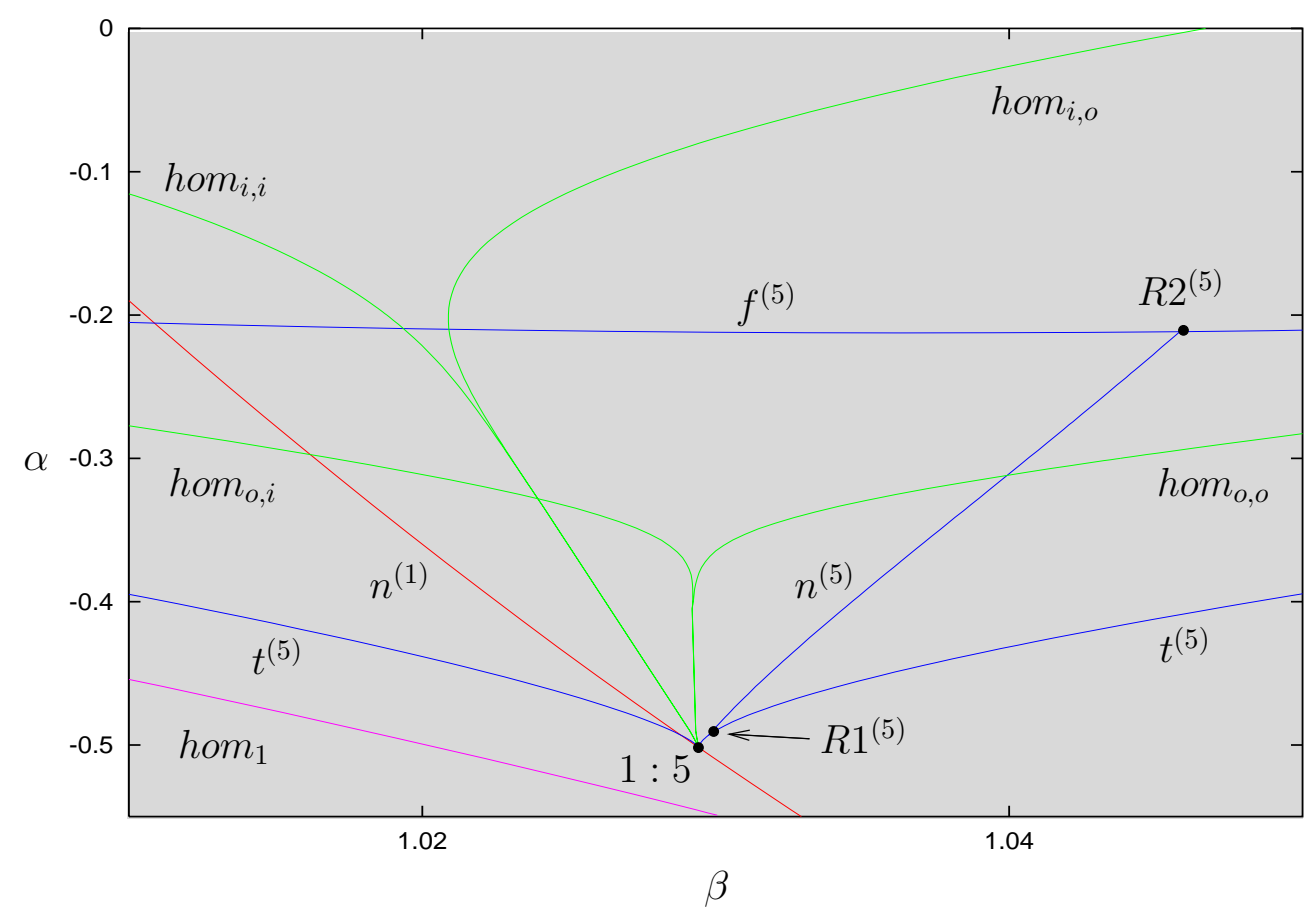

FIG. 3.7. Resonance horn of period 5 for $R=-0.1$. Displayed are curves of Neimark-Sacker(red) and homoclinic tangencies(pink) of period 1. Then for period 5 fold, flip and Neimark-Sacker curves(blue), and homoclinic tangencies(green). The first index i/o refers to the position of the closed curve relative to the saddles. The second index refers to the type of tangency.

on, the curves hom ${ }_{o, i}$ and $h_{o m}, o$ bound another region with homoclinic tangle. If we move further across hom $_{o, o}$ we will again see an invariant circle. Other weak resonances are present and the previous structure repeats itself again. This agrees well with the theoretical picture. However, we note that the model map studied in [1], has some degeneracies, so that only two of four possible branches of homoclinic tangencies appeared. Also the intersection of $h_{o m}$ o with hom ${ }_{o, i}$ may occur either above or below the flip bifurcation curve.

Tracing the Neimark-Sacker curve, we encounter other strong resonances. The 1:4 resonance can have several bifurcation sequences, but when $R$ is small, only case I for positive $R$ and case VIII 


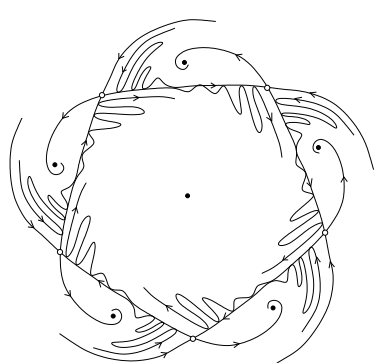

hom $_{i, i}$

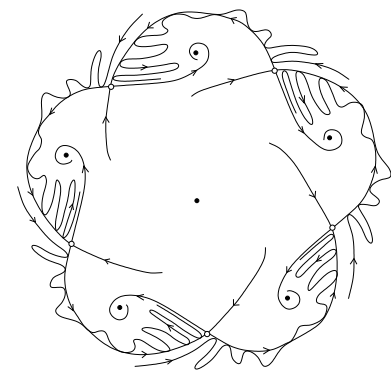

$\operatorname{hom}_{o, i}$
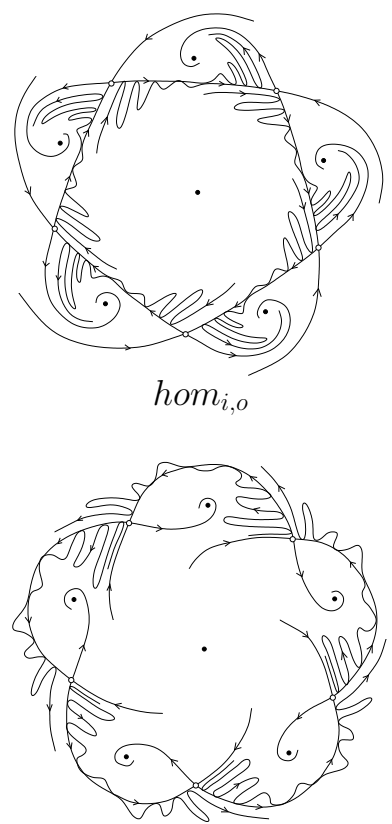

$\operatorname{hom}_{O, o}$

FIG. 3.8. Sketches of various homoclinic tangencies inside the resonance tongue of period 5.

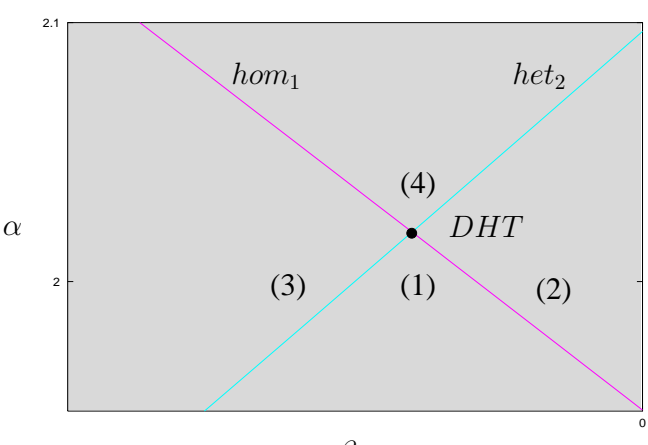

(a)

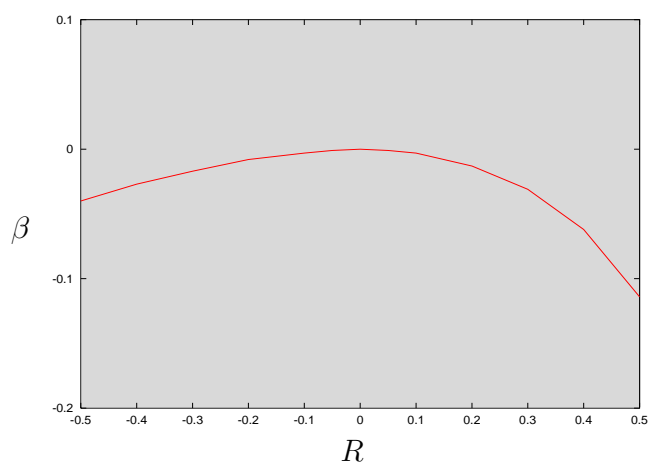

(b)

FIG. 3.9. (a) Close up of the bifurcation diagram of the generalized Hénon map for $R=-0.5$ near the DHT point. Only the primary bifurcations are displayed; (b) Dependence of the $\beta$-coordinate of the DHT point on $R$.

for negative $R$ remain. While case I is the simplest, case VIII has the richest bifurcation sequence (see, for example, [21]). Then the 1:3 resonance is encountered. Apart from a period 3 orbit with a homoclinic connection nothing special occurs. Then finally we meet the 1:2 resonance. For small $R$, this involves only one of two possible cases [21], namely, the one from which the NeimarkSacker curve $n^{(2)}$ for the period-2 cycle emanates. The behavior of the generated invariant circle is determined by the sign of $R$. Continuing further we observe a cascade of nondegenerate 1:2 resonances $R 2^{(k)}$ located on the flip curves and connected by Neimark-Sacker bifurcation segments (in Figure 3.2 only $R 2^{(1)}$ and $R 2^{(2)}$ are visible).

We now give more details on global bifurcations. Above the period-doubling curve there always exists a transversal heteroclinic orbit connecting the two period-1 saddles. The other invariant manifolds of these saddles can also intersect or become tangent. The wedges of the corresponding heteroclinic and homoclinic tangencies (delimited by het h,2 $_{1,2}$ and hom $_{1,2}$ ) form boundaries of parameter regions where nontrivial hyperbolic sets exist. The boundary curves het 2 and hom $_{1}$ 


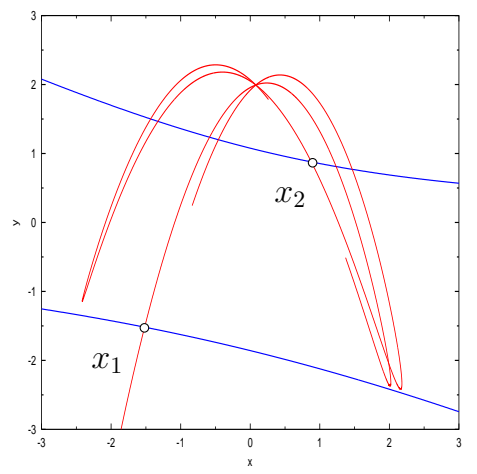

(1)

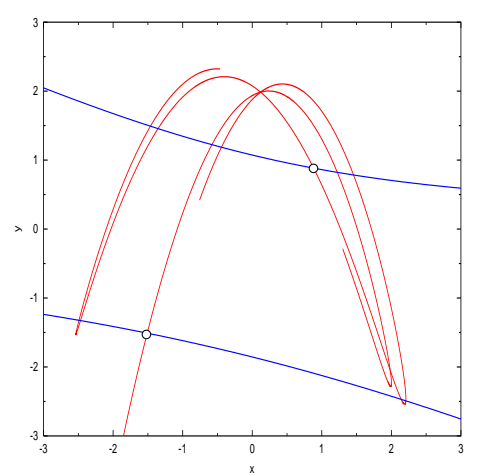

(3)

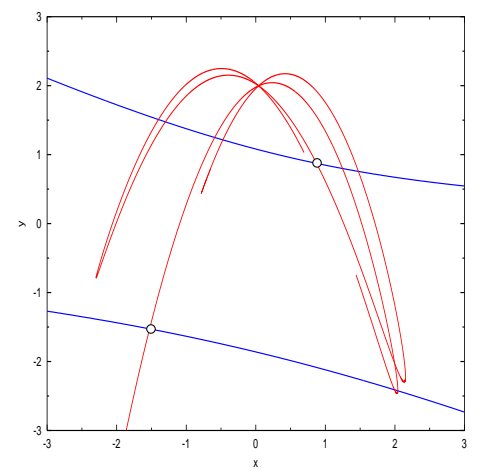

(2)

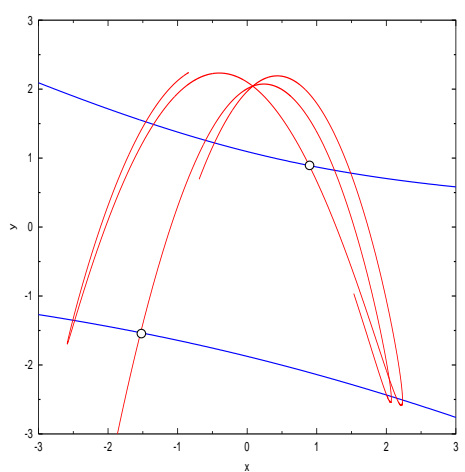

(4)

Fig. 3.10. Stable(blue) and unstable(red) manifolds near the DHT-point. Parameter values are $(1):(\alpha, \beta)=$ $(-0.04,2),(2):(\alpha, \beta)=(-0.02,2),(3):(\alpha, \beta)=(-0.06,2)$ and $(4):(\alpha, \beta)=(-0.04,2.05)$

intersect giving rise to a Double (Homoclinic/Heteroclinic) Tangency $(D H T)$ point, see Figures 3.9 and 3.10. We start in region (1) where there are two saddle fixed points and two transversal heteroclinic orbits which are always present. Going to region (2) the stable manifold of $x_{1}$ becomes tangent to the unstable manifolds of this point and two transversal homoclinic orbits are born. From (1) to (3) the unstable manifolds of $x_{2}$ touch the stable of $x_{1}$ and two heteroclinics are born. In region (4) all six transversal orbits are present.

Finally, let us point out that there exists a homoclinic tangency to a neutral saddle in the generalized Hénon map (see points NHT in Figure 3.11). This means that GHM, which itself was derived to study the homoclinic tangency to a neutral saddle, also exhibits this codim 2 bifurcation. It implies the existence of a fractal bifurcation set near this singularity in the parameter plane. In particular, we see that the fold and flip curves accumulate on the homoclinic tangency curve, while strong 1:1 resonances (slowly) approach the corresponding $N H T$ points.

4. Interpretation. Here we discuss the correspondence between the obtained bifurcation diagrams of the generalized Hénon map and two bifurcations of diffeomorphisms with codim 2 homoclinic tangencies.

4.1. Homoclinic tangency of a neutral saddle in the plane. We begin with a note on invertibility. The standard Hénon map has the line $\beta=0$ in the parameter plane, which corresponds to zero Jacobian (i.e., the map is noninvertible). The GHM has no such line in the parameter plane, but in the phase plane it always has a line of non-invertibility for all $\alpha, \beta$. This line $y=\frac{\beta}{R \lambda^{k}}=\frac{-b c}{R} \gamma^{k}$ shifts to infinity as $k \rightarrow \infty$. The non-invertibility effects are, therefore, inaccessible (not observable) in the original map $f_{\mu}$ near the codim 2 point (when $k$ is sufficiently large), which is consistent with the fact that $f_{\mu}$ is a diffeomorphism. 


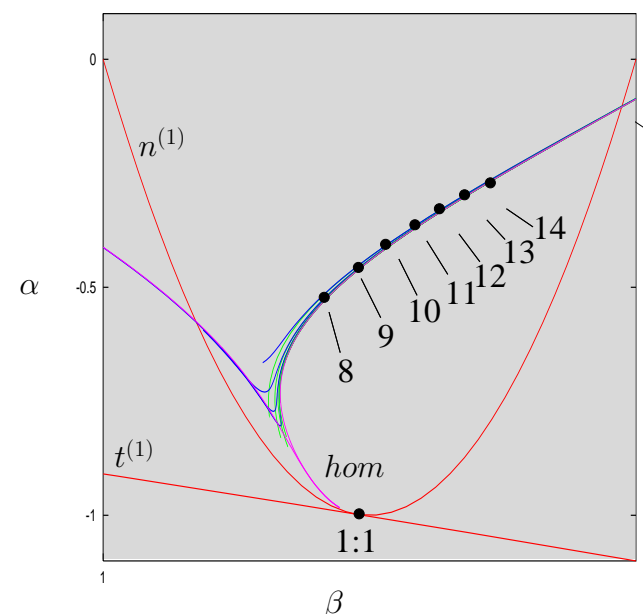

(a)

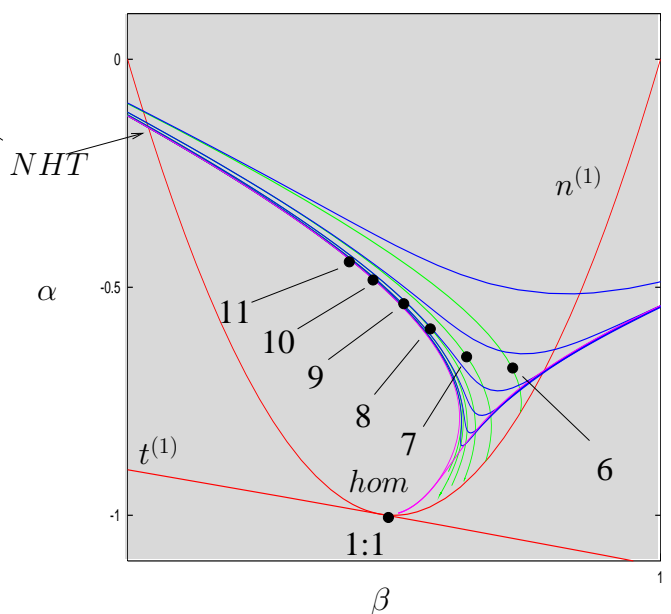

(b)

FIG. 3.11. Resonance 1:1 points near a homoclinic tangency for (a) $R=-0.1$, (b) $R=0.1$, indicated by dots. Resonance 1:2 points are not shown since these lie very close to the 1:1 points. The Neimark-Sacker curves connecting 1:1 and 1:2 resonances are not visible by the same reason.

The correspondence between parameters $(\alpha, \beta)$ and $\left(\mu_{1}, \mu_{2}\right)$ is given by the formulas $(2.4)$ from Section 2. From the second equation in (2.4) follows that in this case $\mu_{2}$ has the asymptotic form

$$
\mu_{2}=\left(-\frac{\beta}{b c}\right)^{\frac{1}{k}}-1
$$

for $k \rightarrow \infty$. Thus, in the $\left(\mu_{2}, \mu_{1}\right)$-plane we can see only one half of the $(\alpha, \beta)$-plane of the GHM, depending on the sign of $b c$ which is determined by the global map: $b c<0$ if the global map is orientation-preserving, $b c>0$ if the global map is orientation-reversing.

4.1.1. Interpretation for fixed $k$. The relationships (2.4) imply that bifurcations which occur in GHM at finite values of parameters $(\alpha, \beta)$ are present in the original map (even infinitely many times - for every $k \geq \bar{k}$ ) and that the corresponding parameter values $\left(\mu_{1}, \mu_{2}\right)$ tend to the point $(0,0)$ as $k \rightarrow \infty$. Moreover here we have infinitely many regions in the $\left(\mu_{1}, \mu_{2}\right)$-plane, where the bifurcation diagram of the GHM appears in the rescaled coordinates. All these regions accumulate on the line $\mu_{1}=0$.

For all $k \geq \bar{k}$ we have the same (rescaled) picture - a "half" of the bifurcation diagram of the generalized Hénon map that is described in the previous section. Note that in the considered case the stability type is the same for fixed points of GHM as for the corresponding orbits in the original planar map. A fixed point of GHM corresponds to a single-round periodic orbit with period $k+n_{0}$ of the original map. A cycle of period $m$ corresponds to a cycle of period $m\left(k+n_{0}\right)$, more precisely to a $m$-round orbit of period $m\left(k+n_{0}\right)$, which exactly $m$ times did $k$ iterations near the saddle (from strip $\sigma_{k}^{0}$ to $\sigma_{k}^{1}$ ) and $n_{0}$ iterations along the "global" part of homoclinic trajectory (from strip $\sigma_{k}^{1}$ to $\sigma_{k}^{0}$ ). A closed invariant curve in GHM corresponds to a closed invariant curve of the $k+n_{0}$-iterate of the original map (in general, it has $k+n_{0}$ disjoint components). The parameters $\alpha, \beta$ on the curves of homoclinic and heteroclinic tangencies in GHM correspond to the tangencies of some one-dimensional manifolds of $k+n_{0}$-periodic orbits.

4.1.2. Interpretation for all periods. If the original diffeomorphism $f_{\mu}$ is orientationpreserving, then $\lambda \gamma>0$ and the global map $T_{1}$ also preserves the orientation. In this case we have the accumulation of the fold bifurcation curves $t_{k}^{(1)}$, the flip bifurcation curves $f_{k}^{(1)}$, and the Neimark-Sacker bifurcation curves $n_{k}^{(1)}$, as well as domains of existence of closed invariant curves, to the line $\mu_{1}=0[9]$. We denote the strips between the fold and flip curves by $D_{k}$. 
These strips also accumulate on the line $\mu_{1}=0$. Moreover, we also have the accumulation of curves of homoclinic tangencies and curves of heteroclinic tangencies of the invariant manifolds of single-round periodic orbits of saddle type.

When $\gamma>0$, the accumulation on the line $\mu_{1}=0$ is monotone (see Figure 4.1(a)).

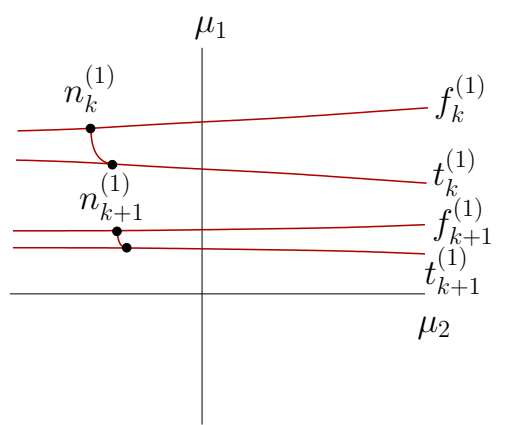

(a)

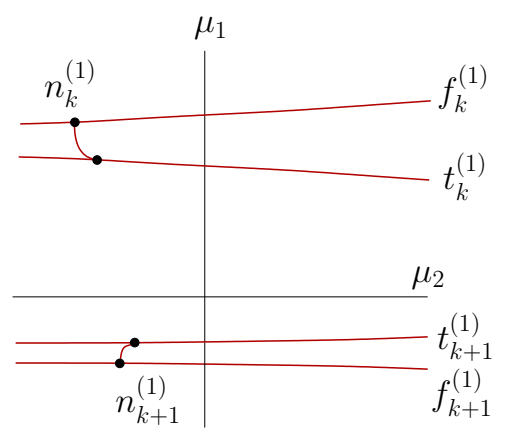

(b)

FIG. 4.1. Bifurcation curves near the planar neutral tangency when the global map is orientation-preserving.

When $\gamma<0$, the accumulation is non-monotone, so that the curves are located above or below of the line $\mu_{1}=0$ depending on the parity of $k$ (see Figure $\left.4.1(\mathrm{~b})\right)^{5}$.

If the original diffeomorphism $f_{\mu}$ is orientation-reversing, then $\lambda \gamma<0$ and the global map $T_{1}$ can either preserve or reverse the orientation, depending on the parity of $n_{0}$. If $T_{1}$ preserves the orientation, we get the described above accumulation of the bifurcation curves $t_{k}^{(1)}$ and $f_{k}^{(1)}$ connected by $n_{k}^{(1)}$. If $T_{1}$ reverses the orientation, we will see no curves $n_{k}^{(1)}$ but, instead, the accumulation of the curves $t_{k}^{(1)}$ and $f_{k}^{(1)}$ touching at the fold-flip points, as $k \rightarrow \infty$ (see Figure 4.2). As above, this accumulation is monotone (Figure 4.2(a)) or non-monotone (Figure 4.2(b)),

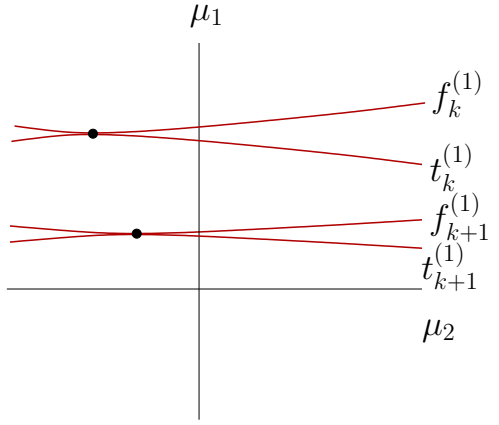

(a)

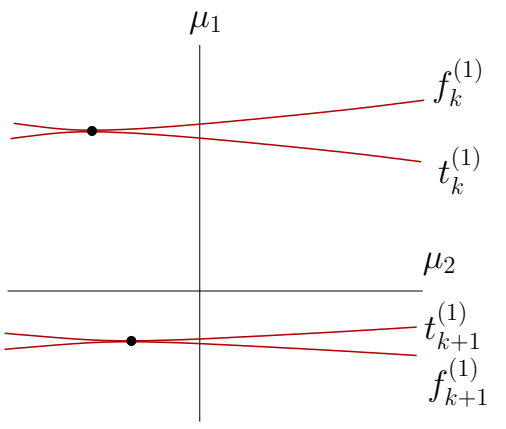

(b)

FIG. 4.2. Bifurcation curves near a planar neutral tangency when the global map is orientation-reversing.

depending on the sign of $\gamma$. Therefore, the whole bifurcation diagram in the case when $f_{\mu}$ reverses the orientation is a superposition of those sketched in Figures 4.1 and 4.2.

4.2. Generalized homoclinic tangency in $\mathbb{R}^{3}$. The correspondence between parameters $(\alpha, \beta)$ and $\left(\mu_{1}, \mu_{2}\right)$ is given in this case by (2.8) from Section 2. Contrary to the planar case, the whole $(\alpha, \beta)$-plane is projected to $\left(\mu_{1}, \mu_{2}\right)$ in this case. Indeed, from the second equation in $(2.8)$

${ }^{5}$ Note that strips in parameter space between $t_{k}^{(1)}$ and $f_{k}^{(1)}$ with different $k$ can intersect when $c>0$. It means, that for some values of parameters (in the intersection of $D_{i}$ and $D_{j}$ ) two single-round periodic orbit of different period can coexist. Moreover, these coexisting orbits can have different type of stability. 
follows the linear asymptotic correspondence between $\mu_{2}$ and $\beta$ in this case, namely

$$
\mu_{2}=-\frac{\beta}{c_{1}\left(\lambda_{1} \gamma\right)^{k}}
$$

for $k \rightarrow \infty$.

Recall that in this case the GHM is obtained via the reduction to a stable two-dimensional invariant center manifold. Therefore a stable point(invariant curve) in GHM corresponds to a stable orbit(invariant curve) of the 3D-diffeomorphism, but a completely unstable point (unstable invariant curve) in GHM corresponds to a saddle orbit (saddle invariant curve) in the original diffeomorphism. Further interpretation is similar to the case of the planar neutral tangency. In particular, the bifurcation curves and regions accumulate on $\mu_{1}=0$. See Figure 4.3 .

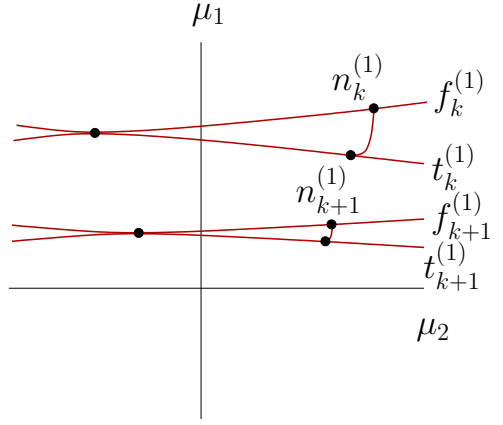

(a)

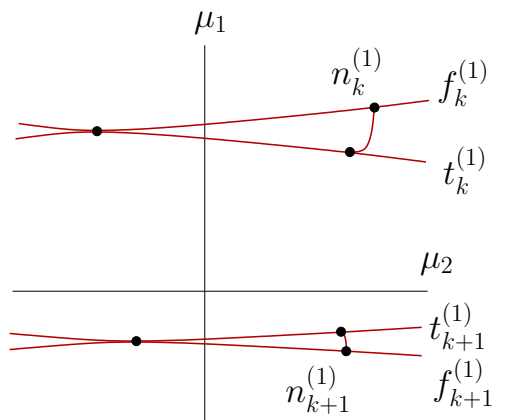

(b)

FIG. 4.3. Accumulation of bifurcation curves in the 3-dimensional case: (a) $\gamma>0$; (b) $\gamma<0$.

5. Discussion. In this paper we gave a rather detailed characterization of the bifurcation structure of the generalized Hénon map (1.3). This allowed us to establish new facts about the codim 2 homoclinic tangencies. To the best of our knowledge, the following results are new:

1. The nondegeneracy of codim 1 and 2 bifurcations of fixed points in (1.3), including all strong resonances, is verified analytically by computing the corresponding normal form coefficients.

2. Accumulation of fold and flip bifurcation curves on heteroclinic tangencies is observed numerically.

3. Cascades of nondegenerate 1:2 resonances of $k$-cycles are found in GHM.

4. It is shown that GHM itself has a homoclinic tangency of a neutral saddle.

5. The above bifurcation phenomena are interpreted in terms of the original maps with the codim 2 homoclinic tangencies. In particular, infinite cascades of homoclinic tangencies of neutral saddles are predicted.

Below we provide some additional comments on these issues.

The accumulation of fold and flip bifurcation curves on the homoclinic tangency curve was first proved in [8] (see Appendix). It is not guaranteed that all fold curves originating from $n^{(1)}$ at weak resonance points have to approach the homoclinic tangency curve. However, the fold curves in GHM (originated at $n^{(1)}$ ) indeed look as if they all approach the corresponding homoclinic tangency curves hom 1 and $h_{0}$. It seems that the $\frac{1}{q}$-resonant periodic orbits are exactly the single-round orbits of period $q$, which exist near homoclinic tangency (see Section 2 for more details). It would be very interesting to explain the correspondence between 2-round (and more round) orbits near the homoclinic tangency and orbits of the original map. There are some topological arguments which do not allow to associate directly $\frac{2}{q}$-resonant orbits with 2 -round periodic orbits.

The accumulation of nondegenerate $1: 2$ resonances $R 2^{(k)}$ is an interesting phenomenon waiting for its analysis. Since its first observation in a periodically forced prey-predator model [24] it has 
been shown to exist in various dynamical systems but the progress in its theoretical understanding is very slow. The generalized Hénon map (1.3) seems to be the most tractable model to study this phenomenon. Indeed, for $S=0$ and small $R$, all 1:2 resonance points are located near the line $\beta=1$, where the standard Hénon map is area-preserving (conservative).

Our numerical analysis demonstrated that the double homoclinic tangency point DHT moves to the half-plane $\beta<0$ for both positive and negative small values of $R \neq 0$ (see Figure $3.9(\mathrm{~b})$ ). This implies that such codim 2 global bifurcations will not be present near the homoclinic tangency to a neutral saddle, if the considered planar diffeomorphism preserves the orientation. Topological reasons for this effect are not clear.

We conclude this discussion with numerical evidence that the resonances 1:1 and 1:2 indeed accumulate to NHT as sketched in Figure 4.1. Note that we see only one half of the parameter plane. Figure 3.11 shows bifurcation curves for orbits with relatively high period together with strong resonances. Figure 3.11 shows that the 1:1 resonance points with periods $\geq 10$ lie practically either on $h_{0 m}$ (a) or on $h_{2}$ (b). However, according to Section 4.2, these resonance points must converge to the NHT point. This is not very evident from the figure, since even the 1:1 points of period 14 (picture (a)) or period 11 (picture (b)) are rather far from the NHT points. Fortunately, this is not a contradiction with the theory. Indeed, from (2.4) in Section 2.1.2 follows that the 1:1 resonance point of period $k$ lies, on the parameter plane, at a distance of order of $O\left(\gamma^{-k}\right)$ from the curve of the homoclinic tangency, where $\gamma$ is the unstable multiplier of the saddle, while the distance from this 1:1 point to the $N H T$ point is only of order $O\left(\frac{1}{k}\right)$. To see this, recall that (4.1) implies that the 1:1 resonance $k$-point has the coordinate $\mu_{2} \sim\left(\frac{1}{-b c}\right)^{\frac{1}{k}}-1 \sim \frac{1}{k} \ln \left(\frac{1}{-b c}\right)$. Thus, such points are exponentially close to the corresponding homoclinic tangency curve but only polynomially close to the NHT point. These two very different asymptotics are clearly visible in Figure 3.11 .

Acknowledgments. The authors are grateful to L.P. Shilnikov and S.V. Gonchenko (Nizhny Novgorod) for useful discussions and to F. Verhulst and M. Ruijgrok (Utrecht) for comments on an early version of the text. V.S.G. was partially supported by the grants: INTAS 2000-221, CRDF-13314, RFBR 02-01-00273 and 01-01-00905.

6. Appendix: Homoclinic bifurcations of codimension 1. We give here a simplified derivation of the well-known results by Gavrilov-Shilnikov [9] using modern rescaling techniques.

Consider a generic one-parameter family $f_{\mu}$ of planar $C^{r}$-diffeomorphisms close to a diffeomorphism $f_{0}$ with a quadratic homoclinic tangency of a saddle fixed point, whose saddle quantity $\sigma$ is assumed to be not equal to one (say, $\sigma<1$ ).

For such families the following classical result is valid [9]: There is a countable set of values $\mu=\mu_{k}^{+}$and $\mu=\mu_{k}^{-}$accumulating on $\mu=0$ as $k \rightarrow \infty$, corresponding to the birth of saddle and stable single-round $\left(k+n_{0}\right)$-periodic orbit at $\mu=\mu_{k}^{+}$and to the period-doubling of such orbit at $\mu=\mu_{k}^{-}$.

As explained in Section 2, the first-return map is constructed as the composition of two maps: global $T_{1}$ and local $T_{0}^{k}$.

For the local map it is known [12] that its $k$ th-iterate $T_{0}^{k}$ can be represented for all sufficiently small $|\mu|$ in the form

$$
\begin{aligned}
& x_{1}=\lambda^{k}(\mu) x_{0}\left(1+\hat{\gamma}^{-k} \xi_{k}\left(x_{0}, y_{1}, \mu\right)\right), \\
& y_{0}=\gamma^{-k}(\mu) y_{1}\left(1+\hat{\gamma}^{-k} \eta_{k}\left(x_{0}, y_{1}, \mu\right)\right),
\end{aligned}
$$

where $\hat{\gamma}=\max \left\{|\lambda(\mu)|^{-1},|\gamma(\mu)|\right\}$ and functions $\xi_{k}$ and $\eta_{k}$ with their derivatives up to and including order $r-2$ are uniformly bounded in $k$, and that $\xi_{k}$ and $\eta_{k}$ with their derivatives up to and including order $r-1$ tend to 0 as $k \rightarrow \infty$. In some sense the iterations of $T_{0}$ are asymptotically close to the iterations of its linear part. Note that the local map is written in the "cross-form" $\left(x_{1}, y_{0}\right) \rightarrow\left(x_{0}, y_{1}\right)$, not as $\left(x_{0}, y_{0}\right) \mapsto\left(x_{1}, y_{1}\right)$. This construction, that can be traced back to Jacobi, is referred to in homoclinic studies as the Neimark-Shilnikov variables. 
The global map $T_{1}$ is taken in the general form

$$
\begin{aligned}
\bar{x}_{0}-x^{+}(\mu) & =G\left(x_{1}, y_{1}, \mu\right), \\
\bar{y}_{0} & =F\left(x_{1}, y_{1}, \mu\right) .
\end{aligned}
$$

Using the geometric properties of the quadratic homoclinic tangency, we can write (6.2) as

$$
\begin{aligned}
\bar{x}_{0}-x^{+} & =a x_{1}+b\left(y_{1}-y^{-}\right)+\cdots \\
\bar{y}_{0} & =\mu+c x_{1}+d\left(y_{1}-y^{-}\right)^{2}+f_{11} x_{1}\left(y_{1}-y^{-}\right)+\cdots,
\end{aligned}
$$

where only essential quadratic terms are displayed ${ }^{6}$. Taking into account (6.1) and (6.3), the first-return map takes the form

$$
\begin{aligned}
\bar{x}_{0} & =x^{+}+a \lambda^{k} x_{0}+b\left(y_{1}-y^{-}\right)+\cdots \\
\gamma^{-k} \bar{y}_{1} & =\mu+c \lambda^{k} x_{0}+f_{11} \lambda^{k} x_{0}\left(y_{1}-y^{-}\right)+d\left(y_{1}-y^{-}\right)^{2}+\cdots .
\end{aligned}
$$

Then, we make the following coordinate shift

$$
x_{0}-x^{+} \mapsto x, \quad y_{1}-y^{-} \mapsto y,
$$

and an additional "small" shift

$$
x \mapsto x+\frac{b f_{11}}{2 d} \lambda^{k}, \quad y+\frac{f_{11} x^{+}}{2 d} \lambda^{k} \mapsto y .
$$

This "kills" the constant term in the first equation and the term linear in $y$ in the second equation, so that (6.4) becomes:

$$
\begin{aligned}
\bar{x} & =b y+\cdots \\
\gamma^{-k} \bar{y} & =\mu-\gamma^{-k} y^{-}+c \lambda^{k} x^{+}+c \lambda^{k} x_{0}+f_{11} \lambda^{k} x y+d y^{2}+\cdots .
\end{aligned}
$$

After the final rescaling

$$
x \mapsto-\frac{b}{d} \gamma^{-k} X, \quad y \mapsto-\frac{1}{d} \gamma^{-k} Y,
$$

(6.7) is transformed into

$$
\left(\begin{array}{c}
X \\
Y
\end{array}\right) \mapsto\left(\begin{array}{c}
Y \\
\alpha+b c(\lambda \gamma)^{k} X-Y^{2}
\end{array}\right)+O\left(\lambda^{k}\right)
$$

where

$$
\alpha=-d \gamma^{2 k}\left(\mu-\gamma^{-k} y^{-}+O\left(\lambda^{k}\right)\right)
$$

When $k \rightarrow \infty$, we obtain the standard Hénon map (1.2) with a small Jacobian $-b c(\lambda \gamma)^{k}($ recall that $\sigma=|\lambda \gamma|<1)$. Note that even though $x$ and $y$ are small, the rescaled variables $X$ and $Y$ cover a large range.

Since the standard Hénon map exhibits the fold and flip bifurcations, infinite sequences of such bifurcations should exist for the family $f_{\mu}$. Indeed, for (6.9), fold and flip bifurcations happen at

$$
\alpha_{k}^{+}=-\frac{1}{4}+O\left(\lambda^{k}\right), \quad \alpha_{k}^{-}=\frac{3}{4}+O\left(\lambda^{k}\right),
$$

respectively (see, for example, Proposition 3.1). Relation (6.10) implies the existence of two infinite sequences of bifurcation parameter values:

$$
\mu_{k}^{+}=\gamma^{-k} y^{-}-\frac{1}{4 d(0)} \gamma^{-2 k}+o\left(\gamma^{-2 k}\right), \quad \mu_{k}^{-}=\gamma^{-k} y^{-}+\frac{3}{4 d(0)} \gamma^{-2 k}+o\left(\gamma^{-2 k}\right),
$$

accumulating on $\mu=0$ as $k \rightarrow \infty$.

\footnotetext{
${ }^{6}$ The dependence of $x^{+}, y^{-}, a, b, c, d$, and $f_{11}$ on $\mu$ is not indicated to simplify notation.
} 


\section{REFERENCES}

[1] V.S. Afraimovich And L.P. Shilnikov, Invariant tori, their break-down and stochasticity, Amer. Math. Soc. Transl, 149, (1991), pp. 201-211. Originally published in: Methods of qualitative theory of differential equations, Gorky State Univ. (1983), pp.3-26.

[2] D.G. Aronson, M.A. Chory, G.R. Hall, And R.P. McGehee, Bifurcations from an invariant circle for two-parameter families of maps of the plane: A computer-assisted study, Comm. Math. Phys., 83 (1982), pp. 303-354.

[3] A. Back, J. Guckenheimer, M. Myers, F. Wicklin, And P. Worfolk, DSTools: Computer assisted exploration of dynamical systems, Notices Amer. Math. Soc., 39 (1992), pp. 303-309.

[4] W.-J. Beyn And J.-M. KleinkAuf, The numerical computation of homoclinic orbits for maps, Siam J. Numer. Anal., 34 (1997), pp. 1207-1236.

[5] V.S. Biragov, Bifurcations in two-parameter family of conservative mappings that are close to the Hénon map, Selecta Math. Sov., 9 (1990), pp. 273-282. Originally published in: Methods of qualitative theory of diff. eqs., Gorky State Univ. (1987), pp. 10-24.

[6] H. Broer, C. Simó, And J.C. TATJer, Towards global models near homoclinic tangencies of dissipative diffeomorphisms, Nonlinearity, 11 (1998), pp. 667-770.

[7] A.R. Champneys, J. Härterich, And B. Sandstede, A non-transverse homoclinic orbit to a saddle-node equilibrium, Ergodic Theory Dynam. Systems, 16 (1996), pp. 431-450.

[8] N.K. Gavrilov AND L.P. Shilnikov, On three-dimensional dynamical systems close to systems with a structurally unstable homoclinic curve - Part 1, Math. USSR Sb., 17 (1972), pp. 467-485.

[9] - On three-dimensional dynamical systems close to systems with a structurally unstable homoclinic curve - Part 2, Math. USSR Sb., 19 (1973), pp. 139-156.

[10] S.V. Gonchenko And V.S. Gonchenko, On Andronov-Hopf bifurcations of two-dimensional diffeomorphisms with homoclinic tangencies. Preprint No. 556, WIAS, Berlin, 2000.

[11] S.V. Gonchenko, V.S. Gonchenko, and J.C. TAtJer, Three-dimensional dissipative diffeomorphisms with codimension two homoclinic tangencies and generalized Hénon maps, in Proc. of Int. Conf. "Progress in Nonlinear Science" dedicated to 100th Anniversary of A.A.Andronov, 2001, pp. 63-79.

[12] S.V. Gonchenko AND L.P. ShILnikov, Invariants of $\Omega$-conjugacy of diffeomorphisms with a structurally unstable homoclinic trajectory, Ukrainian Math. J., 42 (1990), pp. 134-140.

[13] - On two-dimensional area-preserving maps with homoclinic tangencies that have infinitely many generic elliptic periodic points, Notes of St.-Petersburg Steklov Math. Inst., 300 (2003), pp. $155-166$.

[14] S.V. Gonchenko, L.P. Shilnikov, And O.V. Stenkin, On Newhouse regions with infinitely many stable and unstable tori, in Proc. of Int. Conf. "Progress in Nonlinear Science" dedicated to 100th Anniversary of A.A.Andronov, 2002, pp. 80-102.

[15] S.V. Gonchenko, L.P. Shilnikov, And D.V. Turaev, On dynamical properties of diffeomorphisms with homoclinic tangencies. Preprint No. 795, WIAS, Berlin, 2002.

[16] V.S. Gonchenko, On bifurcations of two-dimensional diffeomorphisms with a homoclinic tangency of manifolds of a "neutral" saddle, in Proc. Steklov Inst. Math. 236, 2002, pp. 86-93.

[17] V.S. Gonchenko And S.V. Gonchenko, On bifurcations of birth of closed invariant curves in the case of two-dimensional diffeomorphisms with a homoclinic tangencies, in Proc. of Steklov Math. Inst., 2004. [to appear].

[18] V.S. Gonchenko AND I.I. Ovsyannikov, On bifurcations of three-dimensional diffeomorphisms with a homoclinic tangency to a neutral saddle fixed point, Notes of St.-Petersburg Steklov Math. Inst., 300 (2003), pp. $167-172$.

[19] M. HÉnon, A two-dimensional mapping with a strange attractor, Comm. Math. Phys, 50 (1976), pp. 69-77.

[20] B. Krauskopf And H. Osinga, Growing $1 D$ and quasi-2D unstable manifolds of maps, J. Comp. Physics, 146 (1998), pp. 404-419.

[21] Yu.A. Kuznetsov, Elements of Applied Bifurcation Theory, Springer Verlag, New York, $1995,1998$.

[22] Yu.A. Kuznetsov And V.V. Levitin, CONTENT: A multiplatform environment for analyzing dynamical systems. Dynamical Systems Laboratory, Centrum voor Wiskunde en Informatica, Amsterdam, ftp.cwi.nl/pub/CONTENT, 1997.

[23] Yu.A. Kuznetsov, H.G.E. Meijer, And L. Van. Veen, The fold-flip bifurcation, Int. J. Bifurcation and Chaos, (2004). [to appear].

[24] Yu.A. Kuznetsov, S. Muratori, And S. Rinaldi, Bifurcations and chaos in a periodic predator-prey model, Int. J. Bifurcation and Chaos, 2 (1992), pp. 117-128.

[25] C. MirA, Chaotic Dynamics, World Scientific, Singapore, 1987.

[26] - Some historical aspects of nonlinear dynamics - Possible trends for the future, J. Franklin Inst., 334B (1997), pp. 1075-1113.

[27] D.V. TurAeV, On dimension of non-local bifurcational problems, Int. J. Bifurcation and Chaos, 5 (1996), pp. 919-948. 